\title{
Formation Mechanism of Lath Martensite in Steels*
}

\author{
Yoshinori Murata \\ Department of Materials Design Innovation Engineering, Graduate School of Engineering, Nagoya University, Nagoya 464-8603, Japan
}

Lath martensite formed in low carbon steels plays a crucial role in the mechanical properties of heat-resistant steels containing approximately 0.1 mass\%C. Lath martensite exhibits a hierarchical microstructure comprising packets, blocks and laths. Martensitic transformation is the phase transformation accompanying ordered shear deformation without atom diffusion. The hierarchical microstructure is formed as a result of the relaxation of the strain energy caused by the deformation; however, to the best of our knowledge, the formation mechanism of this microstructure has not been understood thus far. In this paper, the experimental results and phenomenological formation mechanism reported thus far are reviewed, and a new mechanism (including two types of slip deformation (TTSD) model) is introduced, which is constructed by independently considering two kinds of slip deformations using the slip deformation model proposed by Khachaturyan. In addition, the TTSD model allows for the simulation of the martensite phase formation by the phase-field method. Furthermore, the TTSD model permits the prediction of lath martensite features including the existence of sub-blocks and high density of dislocations in lath. In particular, the presence of laths in a block structure is clearly explained by the TTSD model for the first time. [doi:10.2320/matertrans.M2016430]

(Received October 24, 2017; Accepted November 9, 2017; Published January 25, 2018)

Keywords: lath martensite, formation mechanism, phase-field method, dislocation density, lath, block, packet, hierarchical structure, heat-resistant steel

\section{Introduction}

\subsection{Background}

The martensite phase in steels has been investigated since a century as it is responsible for the ever-changing mechanical properties of steels; hence it is crucial for their practical use. Depending on the content of carbon in steel, the morphologies of the martensite phase are classified into plate, lenticular plate and lath ${ }^{1-11)}$. Among these morphologies, this study focuses on lath martensite.

Lath martensite is observed in steels containing less than 1 mass $\% \mathrm{C}$; for example, the cubic martensite structure, in which $c / a$ equals 1 , comprises 0.1 mass $\% \mathrm{C}$ steels, which are crucial heat resistant steels (hereafter, the chemical composition will be referred to as mass\%). Lath martensite is formed in steel with a relatively high Ms temperature. The schematic of the morphology shown in Fig. 1 confirms the hierarchical structure ${ }^{12-17)}$. That is, packets with an approxi-

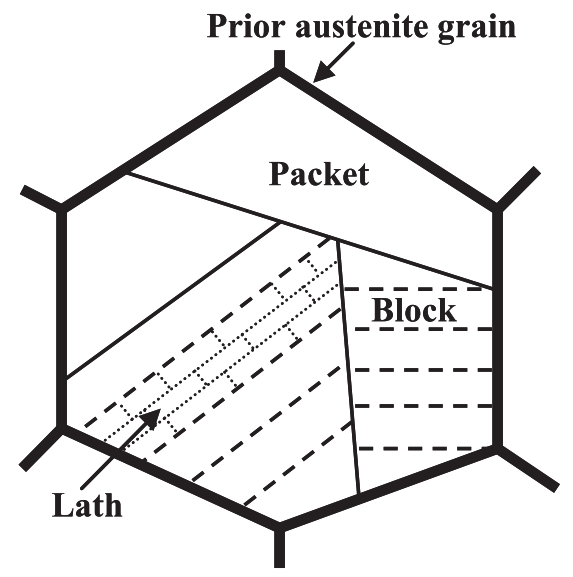

Fig. 1 Schematic of the hierarchical structure of lath martensite.

*This Paper was Originally Published in Japanese in J. Japan Inst. Met. Mater. 80 (2016) 669-683. mately $\{111\}_{\gamma}$ habit plane exist in a prior austenite grain, and a packet comprises strip-shaped blocks aligned parallel to each other. Furthermore, a block comprises laths with similar crystallographic orientations, which contain high-density dislocations with no twin ${ }^{16,17)}$.

As the high-temperature strength of heat-resistant steels depends on their microstructure and on the microstructural change process, it is important to clarify the formation of a hierarchical microstructure and its temporal evolution in steels. In this study, the characteristics of lath martensite are summarized, and studies reported thus far on its formation mechanism are reviewed. Next, a new formation mechanism of lath martensite based on a study recently reported by us, as well as the phenomena predicted by this mechanism is explained. In particular, the presence of laths in a martensite block is clarified.

\subsection{Characteristics of lath martensite}

It is important to summarize the morphological characteristics of the lath martensite reported thus far. Kurdjumov and Sachs have employed X-ray pole figure analysis and reported that the martensite phase exhibits a specific crystallographic relationship with the austenite phase ${ }^{18)}$. Currently, this is called the K-S relationship, comprising $(111)_{\gamma} / /(011)_{\alpha^{\prime}}$ and $[\overline{1} 01]_{\gamma} / /[\overline{1} \overline{1} 1]_{\alpha^{\prime}}$, where $(111)_{\gamma}$ and $(011)_{\alpha^{\prime}}$ represent close-packed planes and $[\overline{1} 01]_{\gamma}$ and $[\overline{1} \overline{1} 1]_{\alpha^{\prime}}$ represent close packed directions in the austenite phase $(\gamma)$ and martensite phase $\left(\alpha^{\prime}\right)$, respectively (in this paper, subscripts $\gamma$ and $\alpha^{\prime}$ denote the austenite and martensite phases, respectively). A total of 24 crystallographic variants satisfies this orientation relationship as shown in Table $1^{11)}$. Besides the K-S relationship, the Nishiyama-Wassermann $(\mathrm{N}-\mathrm{W})$ relationship, i.e., $(111)_{\gamma} / /(011)_{\alpha^{\prime}}$ and $[\overline{2} 11]_{\gamma} / /[01 \overline{1}]_{\alpha^{\prime}}$ has been reported. Furthermore, the Greninger-Troiano (G-T) relationship is the intermediate between the K-S and N-W crystallographic orientation relationships. Among these relationships, lath martensite has been reported to exhibit the K-S relationship ${ }^{14,15,19)}$.

The morphology of the lath has been reported to be a 
plate with $\langle 111\rangle_{\gamma}$ in the longitudinal direction with a ratio of length, width and thickness of approximately 30:7:1 $1^{3,14,15,20)}$, as shown in Fig. 2. In addition, the martensite phase exhibits habit planes near $\{111\}_{\gamma}$; particularly, several studies have reported the $\{557\}_{\gamma}$ habit plane of lath martensite, which deviates by approximately $10^{\circ}$ from the $\{111\}_{\gamma}$ plane $^{21,22)}$.

Recently, the crystal orientation in lath martensite has been analyzed over a wide area using electron back-scattering pattern (EBSP) measurements with scanning electron microscopy (SEM). Prior to the feasibility of EBSP, a block was considered to comprise an assembly of laths with the same variant, which was separated into regions with small crystallographic inclinations to one another; in addition, the inclination boundary was the lath boundary. However, Morito et al. utilized EBSP and reported local deviations in the crystal orientation in low-carbon steels at each point in a block; in addition, they reported that blocks comprised not one but a combination of two specific crystallographic variants ${ }^{16)}$. For example, blocks belonging to variant 4 are observed next to blocks predominantly comprising variant 1 ; similarly, blocks comprising variant 5 are observed next to blocks predominantly comprising variant 2 (see the variant number in Table 1). Morito et al. referred to this morphology as a sub-block structure and distinguished it from conventional blocks ${ }^{16,22)}$.

Martensite blocks are classified into 24 crystallographic variants (Table 1), and a block comprises the aggregation of laths in lath martensite. Note that laths are slightly misoriented within approximately $5^{\circ}$ with respect to each other; hence the aggregation of laths is absolutely different from that of block variants (V1-V24). Meanwhile, studies have not been reported on the reason for the formation of lath aggregation in a block and the main factor for its formation.

\subsection{Previous studies of the formation mechanism of lath martensite}

In the previous section, the experimental results for the crystallographic observation of lath martensite was examined. With respect to theoretical studies, the BowlesMacKenzie (BM) theory and Wechsler-Lieberman-Read (WLR) theory, have been proposed ${ }^{23-26)}$, regarding the deformation geometry of martensitic transformation. The theo- ries are collectively called the phenomenological theory of martensite crystallography (PTMC). PTMC is based on the considerations that the deformation induced by the martensitic transformation should produce an invariant plane because the martensite phase maintains continuity with the surrounding austenite phase. In PTMC, as a transformation sequence, all deformations induced by the martensitic transformation are explained by a combination of lattice deformation (with the change in the crystal structure); lattice-invariant deformation realized by shear deformations; and rigid-body rotation. Sandvik and Wayman ${ }^{27)}$ applied PTMC to lath martensite. However, the above transformation sequence is an artificial one, and it is unlikely that the martensitic transformation actually occurs by the sequence. In other words, PTMC well describes the crystallographic result after the transformation but does not explain the transformation mechanism.

Meanwhile, the two factors deciding the morphology of the martensite phase include nucleation and growth. Olson et al. have reported that lattice defects such as stacking faults and dislocations are crucial for nucleation ${ }^{29,30)}$. Furthermore, Shimizu et al. have suggested that the initial martensite produces other lattice defects in $\mathrm{Fe}-8 \mathrm{Cr}-0.1 \mathrm{C}$ steel; these defects cause a martensite nucleus to have a specific variant (self-catalytic type ${ }^{31)}$ on the basis of the observation of the initial growth of the martensite phase at the stacking faults in the austenite phase.

In contrast to the nucleation of the martensite phase, Khachaturyan have reported a model that describes the de-

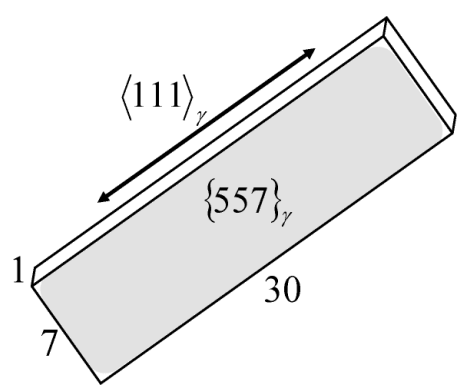

Fig. 2 Schematic of the ratio of dimension of a lath and the crystalline plane.

Table 1 List of 24 variants in the K-S orientation relationship.

\begin{tabular}{|c|c|c|c|c|c|}
\hline Variant No & Close-packed Plane parallel & Close-packed direction parallel & Varian No & Close-packed Plane parallel & Close-packed direction parallel \\
\hline V1 & \multirow{6}{*}{$\begin{array}{c}(111)_{\gamma} / /(011)_{\alpha^{\prime}} \\
\mathrm{CP} 1\end{array}$} & {$[\overline{1} 01]_{\gamma} / /[\overline{1} \overline{1} 1]_{\alpha^{\prime}}$} & V13 & \multirow{6}{*}{$\begin{array}{c}(\overline{1} 11)_{\gamma} / /(011)_{\alpha^{\prime}} \\
\mathrm{CP} 3\end{array}$} & {$[0 \overline{1} 1]_{\gamma} / /[\overline{1} \overline{1} 1]_{\alpha^{\prime}}$} \\
\hline $\mathrm{V} 2$ & & {$[\overline{1} 01]_{\gamma} / /[\overline{1} 1 \overline{1}]_{\alpha^{\prime}}$} & V14 & & {$[0 \overline{1} 1]_{\gamma} / /[\overline{1} 1 \overline{1}]_{\alpha^{\prime}}$} \\
\hline V3 & & {$[01 \overline{1}]_{\gamma} / /[\overline{1} \overline{1} 1]_{\alpha^{\prime}}$} & V15 & & {$[\overline{1} 0 \overline{1}]_{\gamma} / /[\overline{1} \overline{1} 1]_{\alpha^{\prime}}$} \\
\hline $\mathrm{V} 4$ & & {$[01 \overline{1}]_{\gamma} / /[\overline{1} 1 \overline{1}]_{\alpha^{\prime}}$} & V16 & & {$[\overline{1} 0 \overline{1}]_{\gamma} / /[\overline{1} 1 \overline{1}]_{\alpha^{\prime}}$} \\
\hline V5 & & {$[1 \overline{1} 0]_{\gamma} / /[\overline{1} \overline{1} 1]_{\alpha^{\prime}}$} & V17 & & {$[110]_{\gamma} / /[\overline{1} \overline{1} 1]_{\alpha^{\prime}}$} \\
\hline V6 & & {$[1 \overline{1} 0]_{\gamma} / /[\overline{1} 1 \overline{1}]_{\alpha^{\prime}}$} & V18 & & {$[110]_{\gamma} / /[\overline{1} 1 \overline{1}]_{\alpha^{\prime}}$} \\
\hline V7 & \multirow{6}{*}{$\begin{array}{c}(1 \overline{1} 1)_{\gamma} / /(011)_{\alpha^{\prime}} \\
\mathrm{CP} 2\end{array}$} & {$[10 \overline{1}]_{\gamma} / /[\overline{1} \overline{1} 1]_{\alpha^{\prime}}$} & V19 & \multirow{6}{*}{$\begin{array}{c}(11 \overline{1})_{\gamma} / /(011)_{\alpha^{\prime}} \\
\mathrm{CP} 4\end{array}$} & {$[\overline{1} 10]_{\gamma} / /[\overline{1} \overline{1} 1]_{\alpha^{\prime}}$} \\
\hline V8 & & {$[10 \overline{1}]_{\gamma} / /[\overline{1} 1 \overline{1}]_{\alpha^{\prime}}$} & V20 & & {$[\overline{1} 10]_{\gamma} / /[\overline{1} 1 \overline{1}]_{\alpha^{\prime}}$} \\
\hline V9 & & {$[\overline{1} \overline{1} 0]_{\gamma} / /[\overline{1} \overline{1} 1]_{\alpha^{\prime}}$} & V21 & & {$[0 \overline{1} \overline{1}]_{\gamma} / /[\overline{1} \overline{1} 1]_{\alpha^{\prime}}$} \\
\hline V10 & & {$[\overline{1} \overline{1} 0]_{\gamma} / /[\overline{1} 1 \overline{1}]_{\alpha^{\prime}}$} & V22 & & {$[0 \overline{1} \overline{1}]_{\gamma} / /[\overline{1} 1 \overline{1}]_{\alpha^{\prime}}$} \\
\hline V11 & & {$[011]_{\gamma} / /[\overline{1} \overline{1} 1]_{\alpha^{\prime}}$} & $\mathrm{V} 23$ & & {$[101]_{\gamma} / /[\overline{1} \overline{1} 1]_{\alpha^{\prime}}$} \\
\hline V12 & & {$[011]_{\gamma} / /[\overline{1} 1 \overline{1}]_{\alpha^{\prime}}$} & V24 & & {$[101]_{\gamma} / /[\overline{1} 1 \overline{1}]_{\alpha^{\prime}}$} \\
\hline
\end{tabular}


formation in martensitic transformation ${ }^{32)}$ from the viewpoint of the growth of the martensite phase. In this model, all deformations supposedly caused by martensitic transformation are expressed by a combination of lattice deformation (as the crystal structure changes from fcc to bct) and lattice-invariant deformations with plastic deformations by slip. This model is reasonable for the transformation mechanism; however, it has not been verified in detail, and the quantitative analyses were not carried out at the time the theory was proposed.

As mentioned above, several studies have reported the microstructures of the martensite phase; however, the transformation mechanism, as well as the reason for why the lath martensite contains sub-blocks, which was recently reported by EBSD analysis, remains unclear.

For the transformation mechanism, Iwashita et al. proposed a new slip deformation model based on the slip deformation model reported by Khachaturyan. This new model independently considers two types of slip deformations without any adjustable parameter ${ }^{33}$. In the following sections, the details of this model and the new results obtained from it will be discussed.

\section{Formation Model of Lath Martensite by Two Types of Slip Deformations}

\section{$2.1 \quad$ TTSD model}

As mentioned in the previous section, Khachaturyan first reported the formation of martensite by the introduction of slip deformation ${ }^{32)}$; however, he did not provide a reasonable explanation for the formation of the habit plane. On the basis of only the experimental fact that the habit plane of lath martensite is $\{557\}_{\gamma}$, Iwashita et al. proposed a model that can reproduce the habit plane of lath martensite by considering two types of slip deformations ${ }^{33)}$ to release the stress originating from Bain lattice deformation. In this slip model, crystal deformations induced by martensitic transformation are described as a combination of lattice deformation from fcc to bet and plastic deformations to release the strain energy caused by this lattice deformation. Details involving the well-known Bain lattice deformation have been explained below.

First, Bain lattice deformation is described. As shown in Fig. 3(a), a tetragonal unit cell exists between the two fcc unit cells. In this figure, $a_{\gamma}$ represents the lattice parameter of the fcc austenite phase, and $a_{\alpha^{\prime}}$ and $c_{\alpha^{\prime}}$ represent the lattice parameters of the bct martensite phase. The length of the a-axis in the tetragonal unit cell before deformation is $a_{\gamma} / \sqrt{2}$ and the length of the c-axis is $a_{\gamma}$; similarly, the length of the a-axis in the bct unit cell after deformation is $a_{\alpha^{\prime}}$ and that of the c-axis is $c_{\alpha^{\prime}}$. Therefore, the deformation matrix caused by martensitic transformation is expressed below by using $\eta_{1}=\sqrt{2} a_{\alpha^{\prime}} / a_{\gamma}, \eta_{3}=c_{\alpha^{\prime}} / a_{\gamma}$ :

$$
\mathbf{B}_{3}=\left(\begin{array}{ccc}
\eta_{1} & 0 & 0 \\
0 & \eta_{1} & 0 \\
0 & 0 & \eta_{3}
\end{array}\right)
$$

The direction relationship ${ }^{34)}$ between the coordinate systems for the fcc parent phase (austenite phase) and the bct martensite phase is expressed as follows:

$$
\left[\begin{array}{l}
a \\
b \\
c
\end{array}\right]_{\gamma}=\left(\begin{array}{ccc}
1 / 2 & 1 / 2 & 0 \\
-1 / 2 & 1 / 2 & 0 \\
0 & 0 & 1
\end{array}\right)\left[\begin{array}{c}
u \\
v \\
w
\end{array}\right]_{\alpha^{\prime}}
$$

and the plane relationship between the two coordinate systems is expressed as follows:

$$
(h k l)_{\gamma}=(p q r)_{\alpha^{\prime}}\left(\begin{array}{ccc}
1 & -1 & 0 \\
1 & 1 & 0 \\
0 & 0 & 1
\end{array}\right) .
$$

When a bct martensite lattice is formed by Bain deformation (Figs. 3(b) and (c)), the variants are closely related with respect to the crystal orientation as shown in Table 1 . The result provides the two variants V1 and V4. In other words, variants with the minimum deformation formed by $\mathbf{B}_{\mathbf{3}}$ deformation in eq. (1) is V1 or V4.

The relationship described in eqs. (1)-(3), as well as in Figs. 3(b) and (c), is the case in which the $[001]_{\gamma}$ axis coincides with the $[001]_{\alpha^{\prime}}$ axis. In a real crystal, $[100]_{\gamma}$ and $[010]_{\gamma}$ axes possibly coincide with $[001]_{\alpha^{\prime}}$ axis. For the former of the two additional cases, V2 and V5 exhibit the closest crystallographic relationship for the latter V3 and V6 exhibit the closest relationship (Table 1). Figures 4(a)-(c), show the three sets of lattice deformations-V1 and V4, V2 and $\mathrm{V} 5$, and $\mathrm{V} 3$ and $\mathrm{V} 6$, respectively.

For the case in which $[100]_{\gamma}$ coincides with $[001]_{\alpha}$-that is, for the set of V2 and V5 (Fig. 4(b)) - the Bain deformation matrix $\mathbf{B}_{1}$ and the lattice correspondence are expressed as follows:

$$
\mathbf{B}_{1}=\left(\begin{array}{ccc}
\eta_{3} & 0 & 0 \\
0 & \eta_{1} & 0 \\
0 & 0 & \eta_{1}
\end{array}\right)
$$

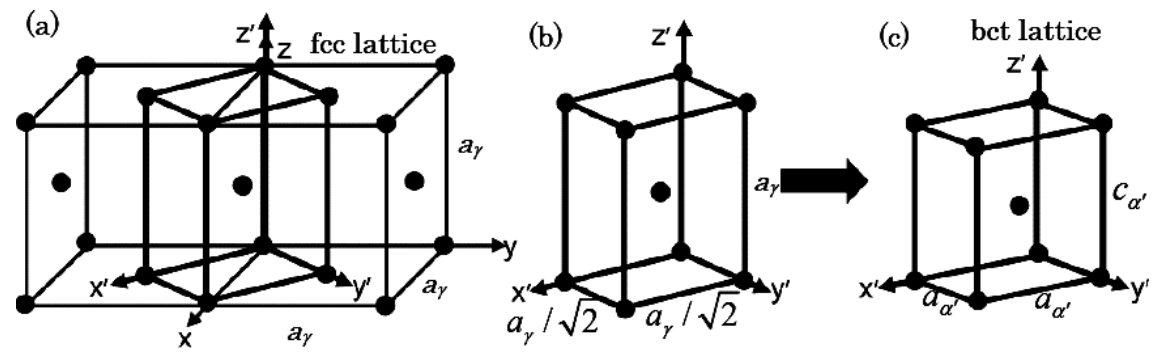

Fig. 3 Illustration of the relationship between the Bain lattice originating from the austenite and martensite lattices. 


$$
\begin{gathered}
{\left[\begin{array}{l}
a \\
b \\
c
\end{array}\right]_{\gamma}=\left(\begin{array}{ccc}
0 & 0 & 1 \\
1 / 2 & 1 / 2 & 0 \\
-1 / 2 & 1 / 2 & 0
\end{array}\right)\left[\begin{array}{c}
u \\
v \\
w
\end{array}\right]_{\alpha^{\prime}},} \\
(h k l)_{\gamma}=(p q r)_{\alpha^{\prime}}\left(\begin{array}{ccc}
0 & 1 & -1 \\
0 & 1 & 1 \\
1 & 0 & 0
\end{array}\right) .
\end{gathered}
$$

For the case in which $[010]_{\gamma}$ coincides with $[001]_{\alpha}$-that is, for the set of V3 and V6 (Fig. 4 (c)) - the Bain deformation matrix $\mathbf{B}_{2}$ and lattice correspondence are expressed as follows:

$$
\begin{gathered}
\mathbf{B}_{2}=\left(\begin{array}{ccc}
\eta_{1} & 0 & 0 \\
0 & \eta_{3} & 0 \\
0 & 0 & \eta_{1}
\end{array}\right), \\
{\left[\begin{array}{l}
a \\
b \\
c
\end{array}\right]_{\gamma}=\left(\begin{array}{ccc}
-1 / 2 & 1 / 2 & 0 \\
0 & 0 & 1 \\
1 / 2 & 1 / 2 & 0
\end{array}\right)\left[\begin{array}{c}
u \\
v \\
w
\end{array}\right]_{\alpha^{\prime}},} \\
(h k l)_{\gamma}=(p q r)_{\alpha^{\prime}}\left(\begin{array}{ccc}
-1 & 0 & 1 \\
1 & 0 & 1 \\
0 & 1 & 0
\end{array}\right) .
\end{gathered}
$$

Here, lath martensite in low-carbon steel is focused, which has a ratio of approximately 1.0 for the lattice constant of $c$ to $a^{35,36)}$; this ratio is considerably less than that of the Bain lattice $(c / a \approx 1.41)$. Thus, the elastically constrained condition can be released by plastic deformations
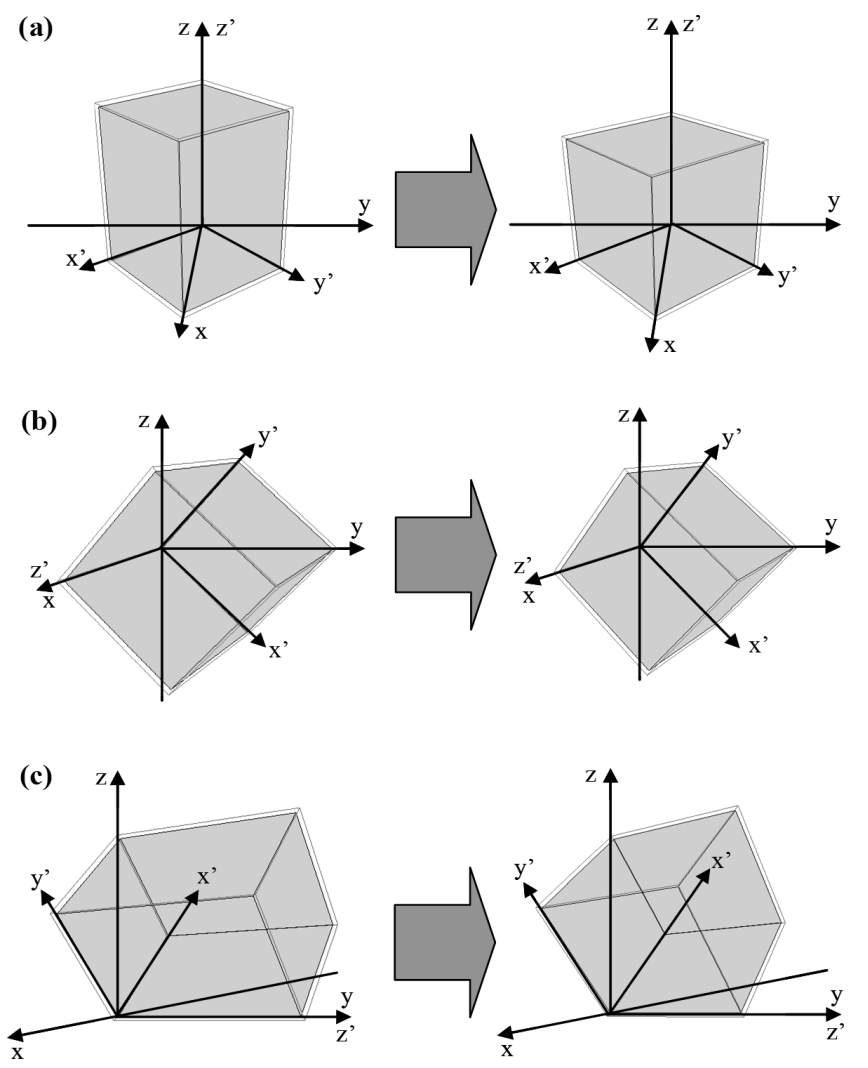

Fig. 4 Illustration of the three types of Bain deformation with respect to the lattice correspondence of $z^{\prime}$ axis of the martensite to the coordinate of the austenite lattice. along the c-axis in the bct martensite lattice. Next, the case in which $[001]_{\gamma}$ axis coincides with $[001]_{\alpha^{\prime}}$ axis is considered (Fig. 3(c)). In the simplest case, this relaxation can be accomplished by two slip systems, [101] $(\overline{1} 01)_{\alpha^{\prime}}$ and $[\overline{101}](101)_{\alpha^{\prime}}$, as shown in Fig. 5(a). Strictly speaking, neither $[101](\overline{1} 01)_{\alpha^{\prime}}$ nor $[\overline{1} 01](101)_{\alpha^{\prime}}$ is a slip system in bct (bcc) crystals, but these slips can be obtained by the combination of two $\mathbf{a} / 2\langle 111\rangle_{\alpha^{\prime}}$ dislocations with the Burgers vectors $\mathbf{b}_{\mathbf{1}}$ and $\mathbf{b}_{\mathbf{2}}$ (Fig. 5(b)). In fact, high-density $\mathbf{a} / 2\langle 111\rangle_{\alpha^{\prime}}$ dislocations have been reported to exist in the lath martensite ${ }^{37)}$. Next, the possibility of the occurrence of the invariant lattice deformation under the above conditions is considered.

With the transformation of the bct martensite crystal vector $\mathbf{r}_{\alpha^{\prime}}$ into $\mathbf{r}_{\alpha^{\prime}}^{\prime}$ by deformation, the relationship between the two vectors is expressed as follows:

$$
\mathbf{r}_{\alpha^{\prime}}^{\prime}=\mathbf{r}_{\alpha^{\prime}}+\mathbf{T}\left(\mathbf{r}_{\alpha^{\prime}}\right)
$$

Here, $\mathbf{T}\left(\mathbf{r}_{\alpha^{\prime}}\right)$ is the total displacement. Because the total displacement is generated by deformations in $[101]_{\alpha^{\prime}}$ and $[\overline{101}]_{\alpha}$ directions, $\mathbf{T}\left(\mathbf{r}_{\alpha^{\prime}}\right)$ can be expressed as follows:

$$
\mathbf{T}\left(\mathbf{r}_{\alpha^{\prime}}\right)=\mathbf{T}_{[101]_{\alpha^{\prime}}} n_{1}+\mathbf{T}_{[\overline{1} 01]_{\alpha^{\prime}}} n_{2} .
$$

Here, $n_{1}$ and $n_{2}$ represent the numbers of active slip planes, and $\mathbf{T}_{[101]_{\alpha^{\prime}}}$ and $\mathbf{T}_{[101]_{\alpha^{\prime}}}$ represent the displacements in each slip direction. In addition, $n_{1}$ and $n_{2}$ can be expressed as follows:

$$
\begin{aligned}
& n_{1}=\frac{1}{m_{1}} \mathbf{H}_{(\overline{1} 01)_{\alpha^{\prime}}} \mathbf{r}_{\alpha^{\prime}}, \\
& n_{2}=\frac{1}{m_{2}} \mathbf{H}_{(101)_{\alpha^{\prime}}} \mathbf{r}_{\alpha^{\prime}},
\end{aligned}
$$

Here, $m_{1}$ and $m_{2}$ represent the average numbers of lattice planes between the nearest active slip plane in each slip system; $\mathbf{H}$ represents the reciprocal lattice vector; and $\mathbf{H}_{(h k)_{\alpha^{\prime}}} \mathbf{r}_{\alpha^{\prime}}$ represents the total number of $(h k l)_{\alpha^{\prime}}$ planes within the length of the vector $\mathbf{r}_{\alpha^{\prime}}$. By substituting eqs. (11)-(13) into eq. (10), we get

$$
\mathbf{r}_{\alpha^{\prime}}^{\prime}=\left(\mathbf{I}+\frac{1}{m_{1}} \mathbf{T}_{[\mathbf{1 0 1}]_{\alpha^{\prime}}} * \mathbf{H}_{(\overline{\mathbf{1} 01})_{\alpha^{\prime}}}+\frac{1}{m_{2}} \mathbf{T}_{[\overline{\mathbf{1} 01}]_{\alpha^{\prime}}} * \mathbf{H}_{(\mathbf{1 0 1})_{\alpha^{\prime}}}\right) \mathbf{r}_{\alpha^{\prime}},
$$

where $\mathbf{I}$ is a unit matrix. In eq. (14), (a)

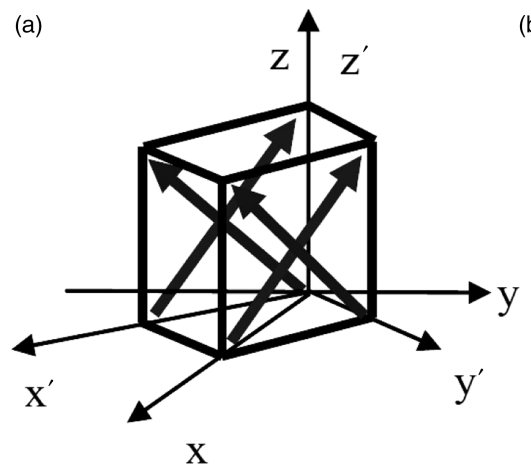

(b)

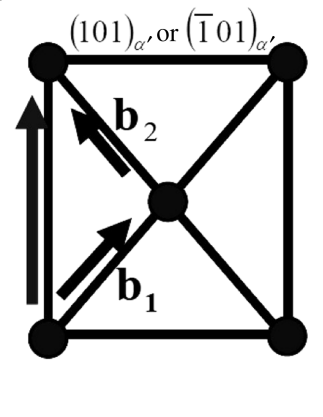

Fig. 5 Illustration of plastic deformations by slips for V1 and V4. 


$$
\left(\mathbf{I}+\frac{1}{m_{1}} \mathbf{T}_{\left[\mathbf{1 0 1}_{\alpha^{\prime}}\right.} * \mathbf{H}_{(\overline{\mathbf{1} 01})_{\alpha^{\prime}}}+\frac{1}{m_{2}} \mathbf{T}_{[\overline{\mathbf{1}} \mathbf{0 1}]_{\alpha^{\prime}}} * \mathbf{H}_{(\mathbf{1 0 1})_{\alpha^{\prime}}}\right)
$$

is a matrix that transforms the vector $\mathbf{r}_{\alpha^{\prime}}$ into $\mathbf{r}_{\alpha^{\prime}}^{\prime}$. In other words, eq. (15) is a matrix representing the plastic deformations.

Thus, the total deformation that occurs by martensitic transformation can be evaluated by the sum of the lattice deformation according to Bain deformation and plastic deformations for releasing the strain caused by the lattice deformation mentioned above. The procedure is explained below.

The transformation of vector $\mathbf{r}_{\gamma}$ (austenite phase) into $\mathbf{r}_{\alpha^{\prime}}$ by lattice deformation is expressed as follows (eq. (1)):

$$
\mathbf{r}_{\alpha^{\prime}}=\mathbf{B}_{\mathbf{3}} \mathbf{r}_{\gamma} .
$$

The substitution of eq. (16) into eq. (14) gives

$\mathbf{r}_{\alpha^{\prime}}^{\prime}=\left(\mathbf{I B} \mathbf{3}_{3}+\frac{1}{m_{1}} \mathbf{T}_{[\mathbf{1 0 1}]_{\alpha^{\prime}}} * \mathbf{H}_{(\overline{\mathbf{1} 01})_{\alpha^{\prime}}} \mathbf{B}_{\mathbf{3}}+\frac{1}{m_{2}} \mathbf{T}_{[\overline{\mathbf{1} 01}]_{\alpha^{\prime}}} * \mathbf{H}_{(\mathbf{1 0 1})_{\alpha^{\prime}}} \mathbf{B}_{\mathbf{3}}\right) \mathbf{r}_{\gamma}$

The total displacement along $[101]_{\alpha^{\prime}}$ using the total displacement along $\left[\frac{1}{2} \frac{\overline{1}}{2} 1\right]_{\gamma}$ and that along $[\overline{1} 01]_{\alpha^{\prime}}$ using the total displacement along $\left[\frac{1}{2} \frac{1}{2} 1\right]_{\gamma}$ can be expressed using eq. (2). Thus, the following equations can be obtained.

$$
\begin{aligned}
& \mathbf{T}_{[\mathbf{1 0 1}]_{\alpha^{\prime}}}=\mathbf{B}_{\mathbf{3}} \mathbf{T}_{\left[\frac{1}{2} \frac{1}{2} \mathbf{1}\right]_{\gamma}}=\left(\begin{array}{ccc}
\eta_{1} & 0 & 0 \\
0 & \eta_{1} & 0 \\
0 & 0 & \eta_{3}
\end{array}\right)\left(\begin{array}{c}
\frac{a_{\gamma}}{2} \\
-\frac{a_{\gamma}}{2} \\
a_{\gamma}
\end{array}\right)=\left(\begin{array}{c}
\frac{a_{\gamma}}{2} \eta_{1} \\
-\frac{a_{\gamma}}{2} \eta_{1} \\
a_{\gamma} \eta_{3}
\end{array}\right), \\
& \left.\mathbf{T}_{[\overline{\mathbf{1}} \mathbf{0} 1]_{\alpha^{\prime}}}=\mathbf{B}_{\mathbf{3}} \mathbf{T}_{\left[\frac{1}{2} \frac{1}{2} 1\right.} \mathbf{1}\right]_{\gamma}=\left(\begin{array}{ccc}
\eta_{1} & 0 & 0 \\
0 & \eta_{1} & 0 \\
0 & 0 & \eta_{3}
\end{array}\right)\left(\begin{array}{c}
-\frac{a_{\gamma}}{2} \\
\frac{a_{\gamma}}{2} \\
a_{\gamma}
\end{array}\right)=\left(\begin{array}{c}
-\frac{a_{\gamma}}{2} \eta_{1} \\
\frac{a_{\gamma}}{2} \eta_{1} \\
a_{\gamma} \eta_{3}
\end{array}\right) .
\end{aligned}
$$

From the relationship

$$
\mathbf{H}_{(\mathbf{p q r})_{\alpha^{\prime}}}=\mathbf{H}_{(\mathbf{h k l})_{\gamma}} \mathbf{B}_{\mathbf{3}}^{-\mathbf{1}},
$$

the following equation is obtained:

$$
\mathbf{H}_{(\mathbf{p q r})_{\alpha^{\prime}}} \mathbf{B}_{\mathbf{3}}=\mathbf{H}_{(\mathbf{h k l})_{\gamma}} .
$$

$\mathbf{H}_{\left(\overline{\mathbf{1}}^{101}\right)_{\alpha^{\prime}}} \mathbf{B}_{\mathbf{3}}$ in eq. (17) can be expressed as $\mathbf{H}_{(\overline{\mathbf{1} 11})_{\gamma}}$ using eqs. (3) and (21) and $\mathbf{H}_{(\mathbf{1 0 1})_{\alpha^{\prime}}} \mathbf{B}_{3}$ in eq. (17) can be expressed as $\mathbf{H}_{(\mathbf{1} \overline{1})_{\gamma}}$. Thus, the following equations can be obtained.

$$
\begin{aligned}
& \mathbf{H}_{(\overline{\mathbf{1 0 1}})_{\alpha^{\prime}}} \mathbf{B}_{\mathbf{3}}=\mathbf{H}_{(\overline{\mathbf{1} 11})_{\gamma}}=\left(\begin{array}{c}
-\frac{1}{a_{\gamma}} \\
\frac{1}{a_{\gamma}} \\
\frac{1}{a_{\gamma}}
\end{array}\right), \\
& \mathbf{H}_{(\mathbf{1 0 1})_{\alpha^{\prime}}} \mathbf{B}_{\mathbf{3}}=\mathbf{H}_{(\mathbf{1} \overline{1} \mathbf{1})_{\gamma}}=\left(\begin{array}{c}
\frac{1}{a_{\gamma}} \\
-\frac{1}{a_{\gamma}} \\
\frac{1}{a_{\gamma}}
\end{array}\right) .
\end{aligned}
$$

The substitution of eqs. (18), (19), (22) and (23) into eq. (17) gives

$\mathbf{r}_{\alpha^{\prime}}^{\prime}=\left(\begin{array}{ccc}\left(1-\frac{1}{2 m_{1}}-\frac{1}{2 m_{2}}\right) \eta_{1} & \left(\frac{1}{2 m_{1}}+\frac{1}{2 m_{2}}\right) \eta_{1} & \left(\frac{1}{2 m_{1}}-\frac{1}{2 m_{2}}\right) \eta_{1} \\ \left(\frac{1}{2 m_{1}}+\frac{1}{2 m_{2}}\right) \eta_{1} & \left(1-\frac{1}{2 m_{1}}-\frac{1}{2 m_{2}}\right) \eta_{1} & \left(-\frac{1}{2 m_{1}}+\frac{1}{2 m_{2}}\right) \eta_{1} \\ \left(-\frac{1}{m_{1}}+\frac{1}{m_{2}}\right) \eta_{3} & \left(\frac{1}{m_{1}}-\frac{1}{m_{2}}\right) \eta_{3} & \left(1+\frac{1}{m_{1}}+\frac{1}{m_{2}}\right) \eta_{3}\end{array}\right) \mathbf{r}_{\gamma}$.
Finally,

$$
\left(\begin{array}{ccc}
\left(1-\frac{1}{2 m_{1}}-\frac{1}{2 m_{2}}\right) \eta_{1} & \left(\frac{1}{2 m_{1}}+\frac{1}{2 m_{2}}\right) \eta_{1} & \left(\frac{1}{2 m_{1}}-\frac{1}{2 m_{2}}\right) \eta_{1} \\
\left(\frac{1}{2 m_{1}}+\frac{1}{2 m_{2}}\right) \eta_{1} & \left(1-\frac{1}{2 m_{1}}-\frac{1}{2 m_{2}}\right) \eta_{1} & \left(-\frac{1}{2 m_{1}}+\frac{1}{2 m_{2}}\right) \eta_{1} \\
\left(-\frac{1}{m_{1}}+\frac{1}{m_{2}}\right) \eta_{3} & \left(\frac{1}{m_{1}}-\frac{1}{m_{2}}\right) \eta_{3} & \left(1+\frac{1}{m_{1}}+\frac{1}{m_{2}}\right) \eta_{3}
\end{array}\right)
$$

is a matrix that represents the total deformation caused by lattice and plastic deformations. V1 or V4 can be determined using the deformation expressed by this matrix.

Of the 24 variants shown in Table 1, the groups V1-V6, V7-V12, V13-V18 and V19-24 belonging to each closepacked plane parallel $(\mathrm{CP})$ group are equivalent with respect to the free-energy change by martensitic transformation. Thus, only the CP1 group is treated to calculate the three types of lattice deformations shown in Figs. 4(a)-(c). The deformation matrix for the lattice deformation corresponding to V1 and V4 (Fig. 4(a)) is expressed in eq. (25). Similarly, the matrix for V2 and V5 (Fig. 4(b)) is expressed as follows:

$$
\left(\begin{array}{ccc}
\left(1+\frac{1}{m_{1}}+\frac{1}{m_{2}}\right) \eta_{3} & \left(-\frac{1}{m_{1}}+\frac{1}{m_{2}}\right) \eta_{3} & \left(\frac{1}{m_{1}}-\frac{1}{m_{2}}\right) \eta_{3} \\
\left(\frac{1}{2 m_{1}}-\frac{1}{2 m_{2}}\right) \eta_{1} & \left(1-\frac{1}{2 m_{1}}-\frac{1}{2 m_{2}}\right) \eta_{1} & \left(\frac{1}{2 m_{1}}+\frac{1}{2 m_{2}}\right) \eta_{1} \\
\left(-\frac{1}{2 m_{1}}+\frac{1}{2 m_{2}}\right) \eta_{1} & \left(\frac{1}{2 m_{1}}+\frac{1}{2 m_{2}}\right) \eta_{1} & \left(1-\frac{1}{2 m_{1}}-\frac{1}{2 m_{2}}\right) \eta_{1}
\end{array}\right),
$$

and that for V3 and V6 corresponding to Fig. 4(c) is expressed as follows:

$\left(\begin{array}{ccc}\left(1-\frac{1}{2 m_{1}}-\frac{1}{2 m_{2}}\right) \eta_{1} & \left(-\frac{1}{2 m_{1}}+\frac{1}{2 m_{2}}\right) \eta_{1} & \left(\frac{1}{2 m_{1}}+\frac{1}{2 m_{2}}\right) \eta_{1} \\ \left(\frac{1}{m_{1}}-\frac{1}{m_{2}}\right) \eta_{3} & \left(1+\frac{1}{m_{1}}+\frac{1}{m_{2}}\right) \eta_{3} & \left(-\frac{1}{m_{1}}+\frac{1}{m_{2}}\right) \eta_{3} \\ \left(\frac{1}{2 m_{1}}+\frac{1}{2 m_{2}}\right) \eta_{1} & \left(\frac{1}{2 m_{1}}-\frac{1}{2 m_{2}}\right) \eta_{1} & \left(1-\frac{1}{2 m_{1}}-\frac{1}{2 m_{2}}\right) \eta_{1}\end{array}\right)$.

\subsection{Deviations from the K-S crystal orientation rela- tionship with respect to close-packed planes and close-packed directions}

The transformation matrix describing martensite was derived in the previous section. However, it is necessary to confirm whether this matrix can produce the lattice deformation in actual steels. Hence, to confirm the validity of eqs. (25)-(27), deviations between the calculated invariant plane and habit plane observed in lath martensite steels are considered. In addition, deviations from the K-S crystal orientation relationship are examined with respect to the closepacked planes and close-packed directions. For these procedures, the eigenvalues of eqs. (25)-(27) are required. The case of eq. (25) is described as an example.

The eigenvalues of the matrix in eq. (25) are $\lambda_{1}, \lambda_{2}$ and $\lambda_{3}$ in ascending order; the corresponding eigenvectors are $\mathbf{e}_{\mathbf{1}}, \mathbf{e}_{\mathbf{2}}$ and $\mathbf{e}_{3}$. The condition that the matrix gives an invariant plane deformation is $\lambda_{1}<1, \lambda_{2}=1, \lambda_{3}>1^{33,38)}$. When this condition is satisfied, the normal vector $\mathbf{n}$ of the invariant plane is expressed as follows:

$$
\mathbf{n}=\sqrt{\frac{1-\lambda_{1}^{2}}{\lambda_{3}^{2}-\lambda_{1}^{2}}} \mathbf{e}_{1}+\sqrt{\frac{\lambda_{3}^{2}-1}{\lambda_{3}^{2}-\lambda_{1}^{2}}} \mathbf{e}_{3} .
$$

Suppose that the vector in the strain direction is $\mathbf{l}$ and the amount of strain is $\varepsilon_{0}$. Then, 


$$
\begin{gathered}
\mathbf{I}=-\lambda_{3} \sqrt{\frac{1-\lambda_{1}^{2}}{\lambda_{3}^{2}-\lambda_{1}^{2}}} \mathbf{e}_{1}+\lambda_{1} \sqrt{\frac{\lambda_{3}^{2}-1}{\lambda_{3}^{2}-\lambda_{1}^{2}}} \mathbf{e}_{3}, \\
\varepsilon_{0}=\lambda_{3}-\lambda_{1} .
\end{gathered}
$$

If the transformation matrix shown in eq. (25) is expressed as $\mathbf{A}$, then $\mathbf{A}$ can be expressed as $\mathbf{A}=\mathbf{D B}_{\mathbf{3}}$ by using $\mathbf{D}$ in eq. (15) and $\mathbf{B}_{3}$ in eq. (1). When the eigenvalues satisfy the above condition, $\lambda_{1}<1, \lambda_{2}=1, \lambda_{3}>1$, the invariant plane deformation is described as $\mathbf{A}_{\mathbf{I}}=\mathbf{I}+\varepsilon_{0} \boldsymbol{l} \otimes \mathbf{n}=$ $\mathbf{R}_{\mathbf{I}} \mathbf{D} \mathbf{B}_{\mathbf{3}}{ }^{32)}$. Here, $\mathbf{R}_{\mathbf{I}}$ is a matrix depending on the average number of lattice planes between the nearest active slip plane in each slip system, $m_{1}$ and $m_{2}$. If $\mathbf{A}_{\mathbf{L}}=\mathbf{R}_{\mathbf{I}} \mathbf{B}_{\mathbf{3}}$ is expressed, then the normal vector $\mathbf{H}_{(p q r)_{\alpha^{\prime}}}$ of an arbitrary plane in the reciprocal space and the vector $\mathbf{T}_{[u v w]_{\alpha^{\prime}}}$ of an arbitrary direction in the martensite lattice are expressed as follows ${ }^{39)}$.

$$
\begin{gathered}
\mathbf{H}_{(p q r)_{\alpha^{\prime}}}=\left(\mathbf{A}_{\mathbf{L}}^{-\mathbf{1}}\right)^{T} \mathbf{H}_{(h k l)_{\gamma}}, \\
\mathbf{T}_{[u v w]_{\alpha^{\prime}}}=\mathbf{A}_{\mathbf{L}} \mathbf{T}_{[a b c]_{\gamma}} .
\end{gathered}
$$

The relationship between the $(p q r)_{\alpha^{\prime}}$ and $(h k l)_{\gamma}$ planes satisfies the lattice correspondence in eq. (3), and that between the $[u v w]_{\alpha^{\prime}}$ and $[a b c]_{\gamma}$ directions satisfies the lattice correspondence in eq. (2).

To investigate the reliability of the deformation expressed in eq. (25) under the condition of $1 \leq m_{1}, m_{1} \leq 100$, the angular deviation of the normal vector of the invariant plane in eq. (28) is evaluated from the normal vector of the $\{557\}_{\gamma}$ habit plane reported by experimental observations via the calculation of the scalar products between the two vectors. Similarly, to determine the crystal orientation relationship, the scalar products between the normal vectors of $(111)_{\gamma}$ and $(011)_{\alpha^{\prime}}$ are first calculated using eq. (31). Then, the scalar products between the vectors $[\overline{1} 01]_{\gamma}$ and $[\overline{1} \overline{1} 1]_{\alpha^{\prime}}$ and between $[01 \overline{1}]_{\gamma}$ and $[\overline{1} 1 \overline{1}]_{\alpha^{\prime}}$ are calculated with eq. (32).

Figure 6 shows the result obtained for the relationship between $m_{1}$ and $m_{2}$ under the condition of $\lambda_{2}=1$ when the eigenvalues of $\lambda_{1}<1, \lambda_{2}=1$, and $\lambda_{3}>1$ in eq. (25) are satisfied: $m_{1}$ and $m_{2}$ are dependent on each other under the condition of $\lambda_{2}=1$. Figure 7 plots the angular deviation between the calculated invariant plane (the normal vector $\mathbf{n}$ ) and the observed habit plane (557) $\gamma$ against the $m_{1}$ value. In this figure, the values on the vertical scale correspond to the angular deviation between the calculated invariant plane and $(557)_{\gamma}$ for V1 and V4, (755) $)_{\gamma}$ for V2 and V5, and (575) $)_{\gamma}$ for V3 and V6. When $m_{1}=m_{2}=17.8$, the angular deviation between the calculated invariant plane and the normal vector of the observed habit plane attains the minimum (Figs. 6 and 7), indicating that the displacement expressed by eqs. (18) and (19) occurs for each of the 17.8 lattice planes.

Figure 8 shows the angular deviations from the closedpacked plane with respect to the K-S orientation relationship, i.e., angular deviation between $(111)_{\gamma}$ and $(011)_{\alpha^{\prime}}$ after deformation given by eq. (25). The deviation angle denoted by the black line becomes less than approximately $5^{\circ}$ at $m_{1}>$ 10 , indicating that close-packed plane parallel is almost satisfied in a wide range of $m_{1}$. Here, the angular deviation between $(111)_{\gamma}$ and $(011)_{\alpha^{\prime}}$ attains the minimum value of approximately $2.5^{\circ}$ at $m_{1}=m_{2}=17.8$.

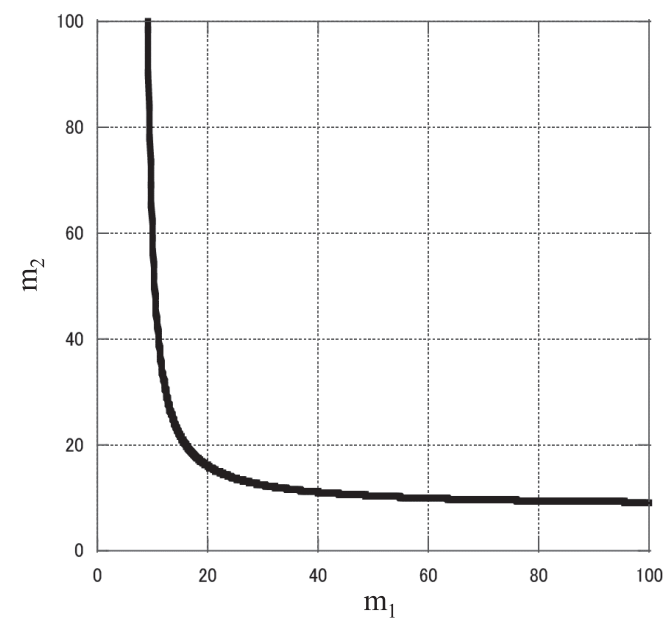

Fig. 6 Calculation result for the relationship between $m_{1}$ and $m_{2}$ values obtained from deformation matrix (eq. (25)), in the presence of the eigenvalues.

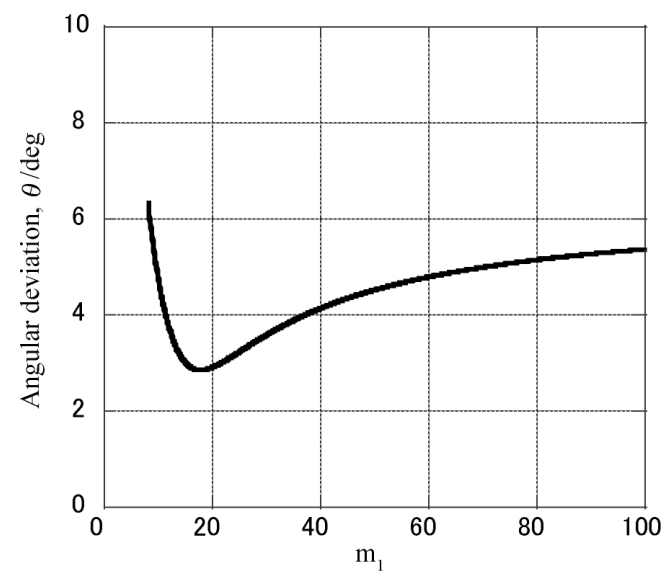

Fig. 7 Angular deviation between the calculated invariant plane and the observed habit plane $(557)_{\gamma}$ versus the $m_{1}$ value.

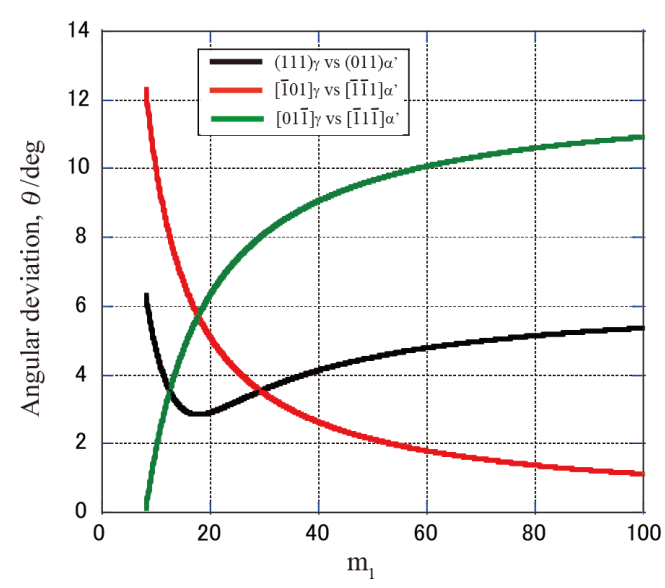

Fig. 8 Angular deviations between $(111)_{\gamma}$ and $(011)_{\alpha^{\prime}}$, between the equivalent close-packed directions $[\overline{1} 01]_{\gamma}$ and $[\overline{1} \overline{1} 1]_{\alpha^{\prime}}$ (for V1), and between the equivalent close-packed directions $[01 \overline{1}]_{\gamma}$ and $[\overline{1} 1 \overline{1}]_{\alpha^{\prime}}$ (for V4), calculated from the deformation matrix (eq. (25)), for V1 and V4. 
The red and green lines in Fig. 8 denote the angular deviation between the equivalent close-packed directions with respect to the K-S orientation relationship. The red line represents the angular deviation between $[\overline{1} 01]_{\gamma}$ and $[\overline{1} \overline{1} 1]_{\alpha^{\prime}}$ for $\mathrm{V} 1$, whereas the green line represents that between $[01 \overline{1}]_{\gamma}$ and $[\overline{1} 1 \overline{1}]_{\alpha^{\prime}}$ for V4. The angular deviations from the closepacked direction with respect to the K-S orientation relationship exhibit a symmetric relationship with respect to $m_{1}$ and $m_{2}$ values when the two variants are produced by the same Bain lattice deformation, e.g., V1 and V4. Here, the angular deviation attains the minimum for $\mathrm{V} 1$ (red line) at $m_{1}=\infty$ and $m_{2} \approx 8$, and for V4 (green line) at $m_{1} \approx 8$ and $m_{2}=\infty$. These results indicate that the $\mathrm{K}-\mathrm{S}$ relationship in the closepacked direction is well satisfied when the deformation is greater in one direction than that in the other. In this case, however, the close-packed plane parallel represented by the black line in Fig. 8 exhibits extreme deviation from the $\mathrm{K}-\mathrm{S}$ relationship. Hence, the plane- and the direction-parallel relationships are not simultaneously satisfied. Thus, to minimize the deviation of the close-packed plain and closepacked direction from the K-S relationship, the $m_{1}$ and $m_{2}$ values should be compromised around 17.8 for minimizing the angular deviation of all three curves in Fig. 8. Hence, lath martensite steels satisfy the relationship of close-packed planes with high accuracy, but deviate by about several degrees from the relationship of close-packed directions, as has been reported previously by experiments ${ }^{40)}$, and the K-S relationship fluctuates by several degrees in each lath, as has been reported thus far ${ }^{41,42)}$.

Only one variant set originates from the same lattice deformation, that is, V1 and V4, but the other variant sets for V2 and V5 and for V3 and V6 can be obtained using the same symmetry relationships with respect to $m_{1}$ and $m_{2}$ (Figs. 7 and 8). Thus, the angular deviations between $[01 \overline{1}]_{\gamma}$ and $[\overline{1} \overline{1} 1]_{\alpha^{\prime}}$ for $\mathrm{V} 3$, and between $[1 \overline{1} 0]_{\gamma}$ and $[\overline{1} \overline{1} 1]_{\alpha^{\prime}}$ for $\mathrm{V} 5$ are the same as $[\overline{1} 01]_{\gamma}$ and $[\overline{1} \overline{1} 1]_{\alpha^{\prime}}$ for V1. Furthermore, the angular deviations between $[\overline{1} 01]_{\gamma}$ and $[\overline{1} 1 \overline{1}]_{\alpha^{\prime}}$ for V2 and between $[1 \overline{1} 0]_{\gamma}$ and $[\overline{1} 1 \overline{1}]_{\alpha^{\prime}}$ for V6 are the same as $[01 \overline{1}]_{\gamma}$ and $[\overline{1} 1 \overline{1}]_{\alpha}$ for V4.

The angular deviations between each calculated invariant plane and the $\{557\}_{\gamma}$ habit plane attain the minimum when $m_{1}=m_{2}=17.8$ (Figs. 6 and 7). Under this condition, the angular deviations from the close-packed direction, which are denoted by red and green lines in Fig. 8, become equal (approximately $5.7^{\circ}$ ), as indicated by the cross-point of the red and green lines in Fig. 8. That is, when the slip deformation occurs under the condition of $m_{1}=m_{2}$, the angular deviation from the close-packed direction for V1 is exactly equal to that for V4, indicating that V1 and V4 are equally present in a martensite crystal. In other words, it is not possible to distinguish these two variants in the $\mathrm{K}-\mathrm{S}$ orientation relationship. Hence, the calculation by Khachaturyan cannot clearly reflect the discussion about variants ${ }^{32}$, in which slip deformation is treated as only $m\left(=m_{1}=m_{2}\right)$. In contrast, if $m_{1}>$ $m_{2}$, a small deviation is observed for V1; in other words, V1 is formed by slip deformation. Similarly, if $m_{2}>m_{1}$, V4 becomes stable. Thus, each variant of lath martensite is determined by the magnitude of the relationship between the two types of plastic deformations, $m_{1}$ and $m_{2}$, after the Bain lattice deformation. For example, V1 is formed by lattice de- formation as shown in Fig. 4(a), and the subsequent plastic deformations primarily comprise the $[101](101)_{\alpha^{\prime}}$ system, whereas V4 comprises the $[\overline{101}](101)_{\alpha^{\prime}}$ system, which may become a new definition for the variant.

Similarly, in Figs. 4(b) and (c), V2 and V3 are characterized by eq. (26) and the $[101](101)_{\alpha^{\prime}}$ system, V5 and V6 by eq. (27) and the $[\overline{101}](101)_{\alpha^{\prime}}$ system.

Note that a variant need not have a unique $m_{i}$ value, indicating that a particular variant is not always formed by the same specific magnitude of plastic deformation and the magnitudes vary widely. This degree of freedom for the $m_{i}$ value permits the existence of a lath (unique $m_{i}$ value) in one variant. In other words, each single lath crystal is a region with a unique $m_{i}$ value and a block is a region with multiple $m_{i}$ values, that is, the aggregation of single laths with slightly different $m_{i}$ values produced by slightly different amounts of dislocations for lattice relaxation. This point will be discussed again in Section 3.4. This result is consistent with the experimental facts that a block is a region comprising the aggregation of single laths with slightly different angles and that sub-blocks are present in the lath martensite reported by Morito et al. ${ }^{16)}$

The TTSD model discussed in Section 2.2 is constructed as an inverse problem on the basis of the experimental facts of the presence of the habit plane. Recently, martensitic transformation has been reportedly analyzed using multiple slip deformations and the coordinate conversion between the transformed regions and the matrix austenite phase by considering the crystal symmetry ${ }^{39)}$. However, the conclusion in the report is essentially the same as that of the TTSD model. The study by Iwashita et al. is cited by L. Qi et al. (Ref. 39) in this paper, but Iwashita's paper is not present in the reference list in the Ref. 39), and an irrelevant paper is cited. Iwashita's paper cited in Ref. 39) is Ref. 33) in this paper.

\section{Experimental Phenomena Clarified by the TTSD Model}

\subsection{Phase-field simulation of the formation of lath mar- tensite based on the TTSD model \\ 3.1.1 Simulation method}

The TTSD model is based on the slip deformation of dislocations to compensate for the lattice strain caused by Bain lattice deformation. This model permits the simulation of the formation of lath martensite because the phase-field (PF) variable representing dislocations has been established as the strain existing around the thin plate obstacle in elastic materials ${ }^{43)}$. A PF model for martensitic transformation can be constructed by the dislocation field introduced by the TTSD model using the PF variables for dislocation, and the transformation sequence can be simulated by the evolution equations of the PF model. The detail is mentioned below.

In the TTSD model, a PF function $\phi_{i}(\mathbf{r})(i=1,2,3)$ is introduced to distinguish the three coordinate coincidences, i.e., the $c$ axis of the tetragonal bct phase is along three equivalent $\langle 100\rangle$ directions in the austenite matrix. Here, $\mathbf{r}$ is the coordinate vector. With respect to plastic deformations, the PF function $p_{i}^{\alpha}(\mathbf{r})(i=1,2,3)$ describes the dislocation slip of the martensite phase, where $\alpha$ is the number of slip systems, and is 1 or 2 in the TTSD model. $p_{i}^{\alpha}$ can be 
evaluated using the following equation.

$$
p_{i}^{\alpha}=\frac{\sqrt{3} \cdot\left|\mathbf{b}_{(i)}\right|}{m_{(i)}^{\alpha} \cdot a_{\gamma}} . \quad(i=1,2,3)
$$

Here, $\mathbf{b}$ is the Burgers vector and $a_{\gamma}$ is the lattice parameter of the austenite phase. $m_{(i)}^{\alpha}$ is the average number of lattice planes between the nearest active slip plane, and has the same meaning as $m$ in eqs. (12), (13), (24), and (25).

The martensitic transformation is a minimization process of the total free energy described by the elasto-plastic PF model. Here, the total free energy $\left(F_{\text {total }}\right)$ is defined as the sum of the chemical energy $E_{c h e m}$, the gradient energy $E_{\text {grad }}$, and the elastic strain, energy $E_{e l}{ }^{43)}$ :

$$
F_{\text {total }}=\int_{\mathbf{r}}\left[f_{L}+E_{\text {grad }_{-} \phi}+E_{\text {grad }_{-} p}+E_{e l}\right] d \mathbf{r} .
$$

Here, $f_{L}$ is a specific local free-energy density, $E_{\text {grad_ } \phi}$ and $E_{\text {grad_p }}$ represent the gradient energy density with respect to the field variables $\phi_{i}(\mathbf{r})$ and $p_{i}^{\alpha}$, respectively, and $E_{\mathrm{el}}$ represents the elastic energy density caused by a coherent interface. $f_{L}$ is expressed by the Landau polynomial expansion of the field variable $\phi_{i}$ as follows ${ }^{44,45)}$ :

$$
f_{L}=\Delta f\left\{\frac{a}{2}\left(\phi_{1}^{2}+\phi_{2}^{2}+\phi_{3}^{2}\right)-\frac{b}{3}\left(\phi_{1}^{3}+\phi_{2}^{3}+\phi_{3}^{3}\right)+\frac{c}{4}\left(\phi_{1}^{2}+\phi_{2}^{2}+\phi_{3}^{2}\right)^{2}\right\} .
$$

Here, $\Delta f$ is the driving force for martensitic transformation, which is equal to the chemical free-energy difference between the austenite and martensite phases. $\Delta f$ is calculated by Thermo-Calc with the CALPHAD method. $a, b$, and $c$ are dimensionless coefficients of the Landau polynomial expansion. In this study, the dimensionless coefficients are chosen as $a=0.1, b=3 a+12$, and $c=2 a+12$ so that $f_{L}$ becomes a local minimum at both $\phi_{i}=0$ and $\phi_{i}=1$.

According to gradient thermodynamics, the gradient energy density $E_{\text {grad_ } \phi}$ accounts for contributions from the spatial variation of $\phi_{i}(\mathbf{r})$, and is represented as ${ }^{46,47)}$

$$
E_{\text {grad_ }_{-}}=\frac{\kappa_{\phi}}{2} \sum_{i=1}^{3}\left(\nabla \phi_{i}(\mathbf{r})\right)^{2}
$$

where $\kappa_{\phi}$ is the gradient energy coefficient with respect to $\phi_{i}(\mathbf{r})$, which is treated as a fitting parameter herein.

The gradient energy density $E_{\text {grad_p }}$ reflects the contribution of the core energy of the dislocations to the plastic-strain accommodation and is expressed as ${ }^{48,49}$ )

$$
E_{\text {grad_p }_{-}}=\frac{\kappa_{p}}{2} \sum_{i=1}^{3} \sum_{\alpha}\left(\left[\mathbf{n}_{(i)}^{\alpha} \times \nabla p_{(i)}^{\alpha}\right] \cdot\left[\mathbf{n}_{(i)}^{\alpha} \times \nabla p_{(i)}^{\alpha}\right]\right),
$$

where $\kappa_{p}$ is the gradient energy coefficient that guarantees the smooth transition of the deformation strain field profile at the interface between the austenite and martensite, and $\mathbf{n}_{i}$ is the unit vector along the slip plane normal.

According to Khachaturyan ${ }^{32)}$, the elastic strain energy is expressed as follows:

$$
E_{e l}=\frac{1}{2} C_{k l m n}\left\{\varepsilon_{k l}(\mathbf{r})-\varepsilon_{k l}^{0}(\mathbf{r})\right\}\left\{\varepsilon_{m n}(\mathbf{r})-\varepsilon_{m n}^{0}(\mathbf{r})\right\},
$$

where $C_{k l m n}$ is the elastic coefficient, which can be expressed as $C_{k l m n}=\lambda \delta_{k l} \delta_{m n}+\mu\left(\delta_{k m} \delta_{l n}+\delta_{k n} \delta_{l m}\right) ; \delta(x)$ is the Dirac delta function; and $\lambda$ and $\mu$ are the Lamé constants, which are estimated from the Young's modulus and the bulk modulus for an isotropic cubic crystal, respectively. $\varepsilon_{k l}(\mathbf{r})$ is the total strain defined as the sum of the homogeneous strain $\bar{\varepsilon}_{k l}$ and the heterogeneous strain $\delta \varepsilon_{k l}$ :

$$
\varepsilon_{k l}(\mathbf{r})=\bar{\varepsilon}_{k l}+\delta \varepsilon_{k l}(\mathbf{r}) .
$$

$\bar{\varepsilon}_{k l}$ describes the macroscopic shape deformation of the system. When the macroscopic shape of the system is fixed during martensitic transformation, the homogeneous strain is zero. This condition is considered to be satisfied in the prior austenite gain boundaries. The heterogeneous strain $\delta \varepsilon_{k l}$ is defined to satisfy $\int_{V} \delta \varepsilon_{k l}=0$. According to the theory of elasticity $^{32)}, \delta \varepsilon_{k l}$ is expressed as follows:

$$
\delta \varepsilon_{k l}(\mathbf{n})=\frac{1}{2}\left\{n_{l} \Omega_{k m}(\mathbf{n})+n_{k} \Omega_{l m}(\mathbf{n})\right\} \sigma_{m n}^{0}(\mathbf{n}) n_{n},
$$

where $\mathbf{n} \equiv \mathbf{k} /|\mathbf{k}|$ is given by the wave number $\mathbf{k}$ in the Fourier space. $\Omega_{i j}(\mathbf{n})$ is the Green function tensor expressed as follows:

$$
\Omega_{i j}(\mathbf{n})=\frac{\delta_{i j}}{\mu}-\frac{n_{i} n_{j}}{2 \mu(1-v)} .
$$

$\delta_{i j}$ is the delta function and $\sigma_{m n}^{0} \equiv C_{k l m n} \varepsilon_{k l}^{0} . \varepsilon_{k l}^{0}(\mathbf{r})$ is the total eigen strain, which is the sum of the elastic strain caused by Bain and plastic deformations. In other words, the elastic strain energy incorporates the contributions of the order parameter $\phi_{i}(\mathbf{r})$ with respect to lattice deformation and $p_{i}^{\alpha}(\mathbf{r})$ with respect to the dislocations. According to Zhou et al. ${ }^{49)}$, the total eigen strain is defined as

$\varepsilon_{k l}^{0}=\sum_{i=1}^{3}\left[\left(\varepsilon_{k l}^{B}(i) \cdot \phi_{i}(\mathbf{r})\right)+\sum_{\alpha} \frac{\mathbf{b}_{(i)}^{\alpha} \otimes \mathbf{n}_{(i)}^{\alpha}+\mathbf{n}_{(i)}^{\alpha} \otimes \mathbf{b}_{(i)}^{\alpha}}{2\left|\mathbf{b}_{(i)}^{\alpha}\right|} \cdot p_{i}^{\alpha}(\mathbf{r})\right]$,

where $\mathbf{b}$ is the Burgers vector, $\mathbf{n}$ is the unit vector of the slip plane normal, and $\otimes$ represents the dyadic product. $\varepsilon_{k l}^{B}(i)$ is the eigenstrain caused by Bain deformation, which is listed in the matrix form as follows:

$$
\begin{gathered}
\varepsilon_{k l}^{B}(1)=\left(\begin{array}{ccc}
\sqrt{2} a_{\alpha^{\prime}} / a_{\gamma}-1 & 0 & 0 \\
0 & \sqrt{2} a_{\alpha^{\prime}} / a_{\gamma}-1 & 0 \\
0 & 0 & c_{\alpha^{\prime}} / a_{\gamma}-1
\end{array}\right), \\
\varepsilon_{k l}^{B}(2)=\left(\begin{array}{ccc}
c_{\alpha^{\prime}} / a_{\gamma}-1 & 0 & 0 \\
0 & \sqrt{2} a_{\alpha^{\prime}} / a_{\gamma}-1 & 0 \\
0 & 0 & \sqrt{2} a_{\alpha^{\prime}} / a_{\gamma}-1
\end{array}\right), \\
\varepsilon_{k l}^{B}(3)=\left(\begin{array}{ccc}
\sqrt{2} a_{\alpha^{\prime}} / a_{\gamma}-1 & 0 & 0 \\
0 & c_{\alpha^{\prime}} / a_{\gamma}-1 & 0 \\
0 & 0 & \sqrt{2} a_{\alpha^{\prime}} / a_{\gamma}-1
\end{array}\right) .
\end{gathered}
$$

By inserting eqs. (39)-(45) into eq. (38), the elastic strain energy is estimated, which in turn can be used to determine the total free energy for martensitic transformation.

The field variables for martensitic transformation are non-conservative ones; hence, the dynamics of the transformation is controlled by the Allen-Cahn equation ${ }^{50)}$.

$$
\frac{\partial M(\mathbf{r}, t)}{\partial t}=-L_{M} \frac{\delta E_{\text {total }}}{\delta M(\mathbf{r}, t)}
$$

where $M(\mathbf{r}, t)\left(M=\phi_{i}, p_{i}^{\alpha}\right)$ indicates the PF functions of the 
coordinate vector $\mathbf{r}$ and evolution time t. $L_{M}$ is the kinetic parameter of each PF parameter. By solving the kinetic equations, martensitic transformation can be simulated by minimizing the total free energy.

\subsubsection{Simulation results ${ }^{51,52)}$}

In this section, as a typical example of the simulation, the three-dimensional simulation results for $\mathrm{Fe}-0.1 \% \mathrm{C}$ steel at $300 \mathrm{~K}$ are discussed. Simulations are performed for a cube with $\mathrm{N}^{3}(\mathrm{~N}=64)$ grid points. The grid size is $4 \mathrm{~nm}$; therefore, the computational domain is $256 \times 256 \times 256 \mathrm{~nm}^{3}$. For the initial state, a plate-like martensite nucleus with a radius of $12 \mathrm{~nm}$ is set on the $(111)_{\gamma}$ plane at the center of a homogeneous austenite crystal. The nucleus comprises a single variant $\mathrm{V} 1$, and the simulation results are confirmed to not depend considerably on the type of nucleus variant. Table 2 summarizes the simulation parameters used. Assuming an isotropic calculation system, the Lamé constants $\lambda$ and $\mu$ are estimated from the Young's modulus and the Bulk modulus for pure iron ${ }^{53)}$. The PF function $\phi_{i}(\mathbf{r})$ changes from 0 to 1 , where 0 and 1 correspond to the austenite and full martensite phases, respectively. The range of $p_{i}^{\alpha}$ is determined to be between 0 and 1.21 , where 0 and 1.21 represent no slip deformation and the maximum slip deformation, respectively.

Figure 9 shows the simulation result for the time evolution of energy densities in Fe- $0.1 \% \mathrm{C}$ steel. The elastic and gradient energy densities monotonically increase and reach a saturated value of approximately $1300 \mathrm{~J} / \mathrm{mol}$ at about 10 time steps, while the chemical free-energy density monotonically decreases with time $t^{*}$ from 0 to $-4600 \mathrm{~J} / \mathrm{mol}$. The chemical free energy is the driving force for martensitic transformation to overcome the increase in the sum of the elastic and gradient energies, leading to the minimization of

Table 2 Simulation parameters for the phase-field model in Fe-0.1C mass $\%$ steel.

\begin{tabular}{ll}
\hline Lattice parameters, $a(\mathrm{~m})$ & $a_{\gamma}=3.599 \times 10^{-10}, a_{\alpha^{\prime}}=2.867 \times 10^{-10}$, \\
& $c_{\alpha^{\prime}}=2.880 \times 10^{-10}$ \\
Elastic constants, $C_{i j k l}(\mathrm{GPa})$ & $C_{11}=267, C_{12}=123, C_{44}=72$ \\
Gradient coefficients, $\left(\mathrm{J} \cdot \mathrm{m}^{2} / \mathrm{mol}\right)$ & $\kappa_{\phi}=1.6 \times 10^{-14}, \kappa_{p}=30 \times 10^{-14}$ \\
Driving force, $\Delta f(\mathrm{~J} / \mathrm{mol})$ & 5085 \\
Temperature, $T(\mathrm{~K})$ & 300 \\
\hline
\end{tabular}

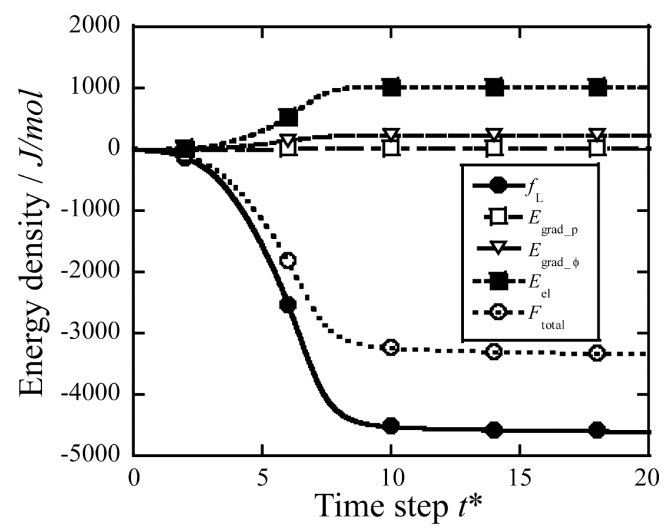

Fig. 9 Simulation result for the time evolution of energy densities in $\mathrm{Fe}$ $0.1 \mathrm{C}$ mass $\%$ steel at $300 \mathrm{~K}$. the total free energy. Note that all energy densities are steady at around 10-15 time steps, indicative of the end of martensitic transformation.

Figure 10 shows the simulation results from the time evolution of three blocks in the CP1 group on the $(111)_{\gamma}$ plane. Dark blue area represents the retained austenite phase and the other colored areas (i.e., red, blue, and yellow areas) represent the three Bain lattice groups (Fig. 4). The occurrence of each domain is determined by the field variable $\phi_{i}(\mathbf{r})$. In this simulation, the martensite phase is assumed to appear only when $\phi_{i}(\mathbf{r}) \geq 0.7$. The three colored domains result from the selectivity of the $c$ axis of the bct martensite phase in the fcc austenite phase. All three blocks are observed at $t^{*}=2$, and different blocks increase in size around the existing martensite phase at $t^{*}=4$ (Fig. 9). At $t^{*}=8$, different domains considerably grow largely along the existing martensite phase. During the growth of martensite, when a second-order block comes in contact with a first-order block, it stops growing. At $t^{*}=20$, the three blocks occupy the whole area, indicative of the accomplishment of the martensitic transformation. The domain formation is independent of the type of the initial nucleus of the martensite, and the domain is spontaneously formed for releasing the large lattice strain caused by the martensitic transformation. In other words, domain is formed as a result of the formation of the martensite phase $\left(\phi_{i}(\mathbf{r})\right)$, and then, the lattice strain is released by plastic strain $\left(p_{i}^{\alpha}(\mathbf{r})\right)$ to decrease the total energy expressed in eq. (34).

Figure 11 shows the simulation results obtained from the time evolution of lath variants in the $\mathrm{CP} 1$ group on the (111) $\gamma$ plane. Six colors corresponding to six variants are observed, i.e., V1-V4, V2-V5, and V3-V6, which is the socalled sub-block structure in lath martensite (Fig. 11(e)).

Figure 12 shows the simulation results obtained from the time evolution of the plastic strain $p_{i}^{\alpha}(\mathbf{r})$ along the slip systems of $[101](101)_{\alpha^{\prime}}$ on the $(111)_{\gamma}$ plane. The dark blue area indicates no slip deformation, while the red area represents the most dramatic slip deformation. This figure reveals that plastic deformation originates from the center of the austenite phase, and the range of slip deformation extends with the progression of martensitic transformation because of the self-relaxation of elastic strain caused by martensitic transformation. A comparison of Figs. 11 and 12 reveals that the morphology of the variant evolution corresponds to that of the plastic strain evolution. With the progression of the phase transition, the plastic strain increases. Hence, the growth and coalescence of the martensitic variants occur by self-relaxation (Fig. 12). In other words, the occurrence and evolution of plastic deformation determine the formation and morphology evolution of the variants in lath martensite.

Figure 13 shows the simulation results obtained from the time evolution of the plastic strain $p_{i}^{\alpha}(\mathbf{r})$ along the slip system of $[101](\overline{101})_{\alpha^{\prime}}$, which is a slip system different from that shown in Fig. 12. A comparison of Figs. 12 and 13 reveals a similar morphology for plastic deformation; however the plastic deformations along the two slip systems are complementary to each other. For example, by considering the area "A" in Fig. 12 and area "B" in Fig. 13, the plastic strain in " $\mathrm{A}$ " is extremely large, while almost no plastic deformation is observed in the same area along the other slip system, 


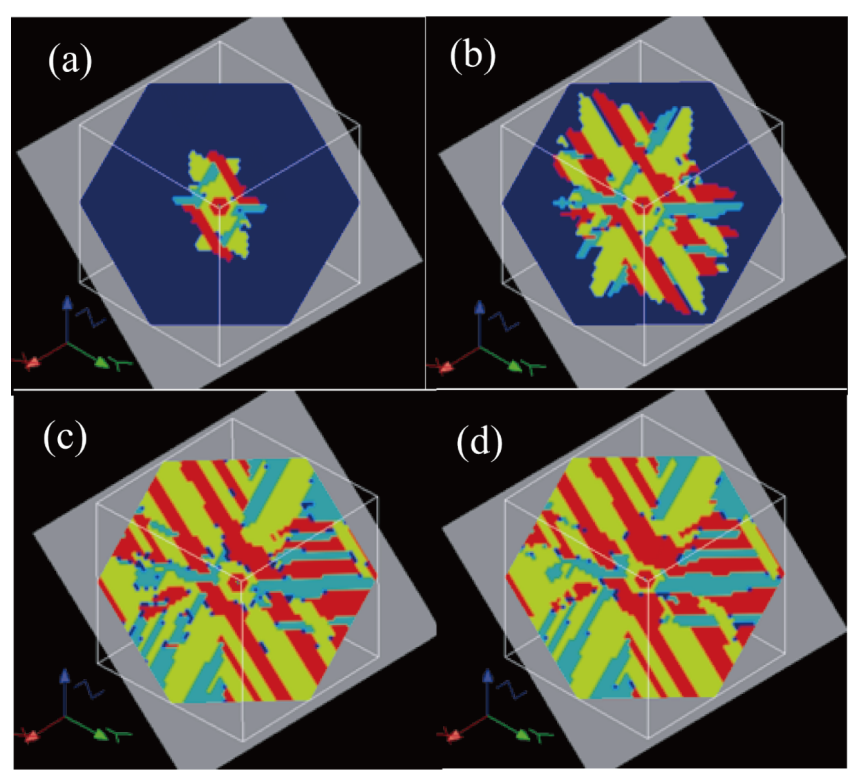

Fig. 10 Time evolution for the blocks of the Bain group on the (111) plane for (a) $t^{*}=2$, (b) $t^{*}=4$, (c) $t^{*}=8$, and (d) $t^{*}=20$ simulated by the elasto-plastic phase-field model. Blue, red, and yellow represent the three Bain groups, while dark blue represents the austenite phase.

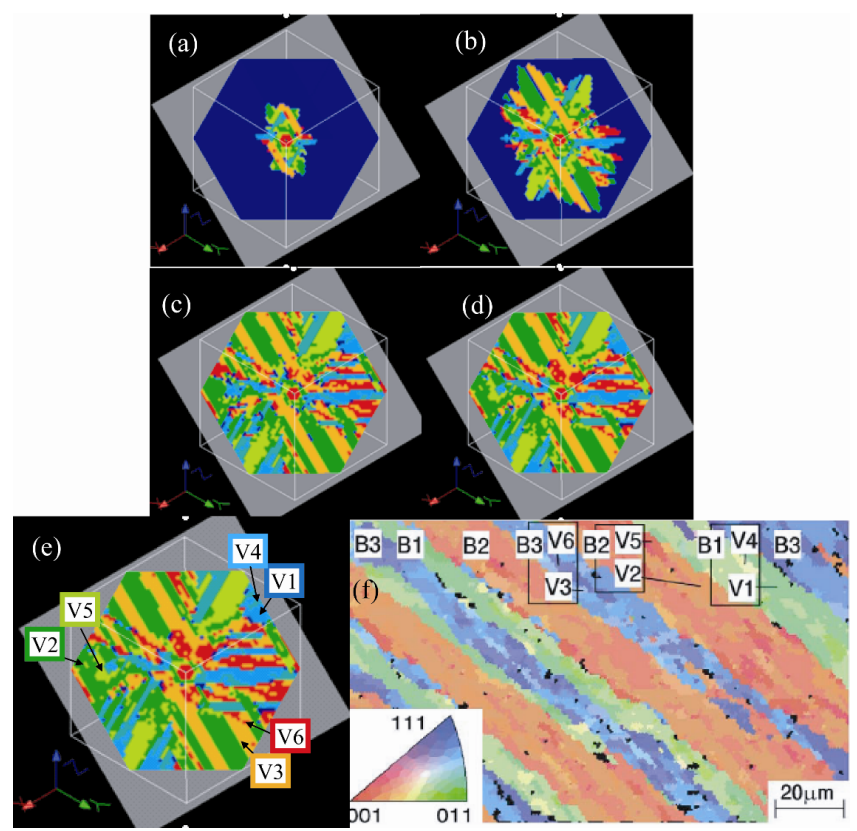

Fig. 11 Time evolution for the variants on the (111) plane for (a) $t^{*}=2$, (b) $t^{*}=4$, (c) $t^{*}=8$, and (d) $t^{*}=20$ simulated by the elasto-plastic phase-field model. (e) Specific explanation of variants shown in (d). Six colors represent six variants in a CP group. (f) EBSP observation of lath martensite quoted from Ref. 16).

as shown in "B". This phenomenon can be observed throughout the martensitic transformation. Hence, the slip deformations along the two slip systems cooperate with each other to assist the plastic strain accommodation.

Figure 14 shows the simulation results obtained from the time evolution of elastic strain energy on the $(111)_{\gamma}$ plane. The red represents the maximum value, and the blue represents the minimum value, i.e., when the elastic strain energy is zero. At an initial stage of martensitic transformation,

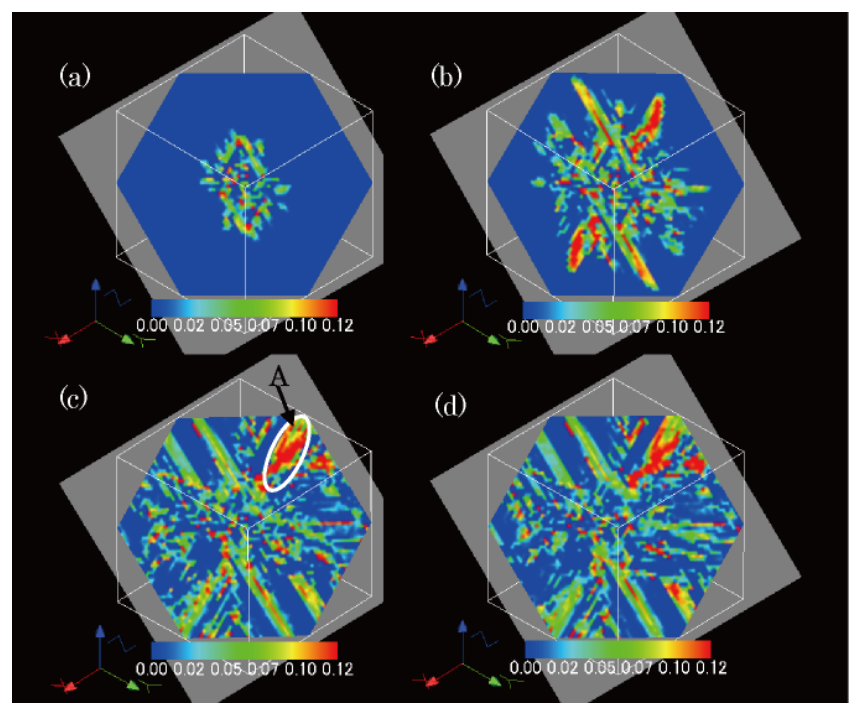

Fig. 12 Time evolution for the plastic strain, $p_{i}^{\alpha}(\mathbf{r}),(i=1,2,3)$, along the $[101](\overline{101})_{\alpha^{\prime}}$ slip system in view of the (111) plane for (a) $t^{*}=2$, (b) $t^{*}=$ 4 , (c) $t^{*}=8$, and (d) $t^{*}=20$ by the phase-field simulation.

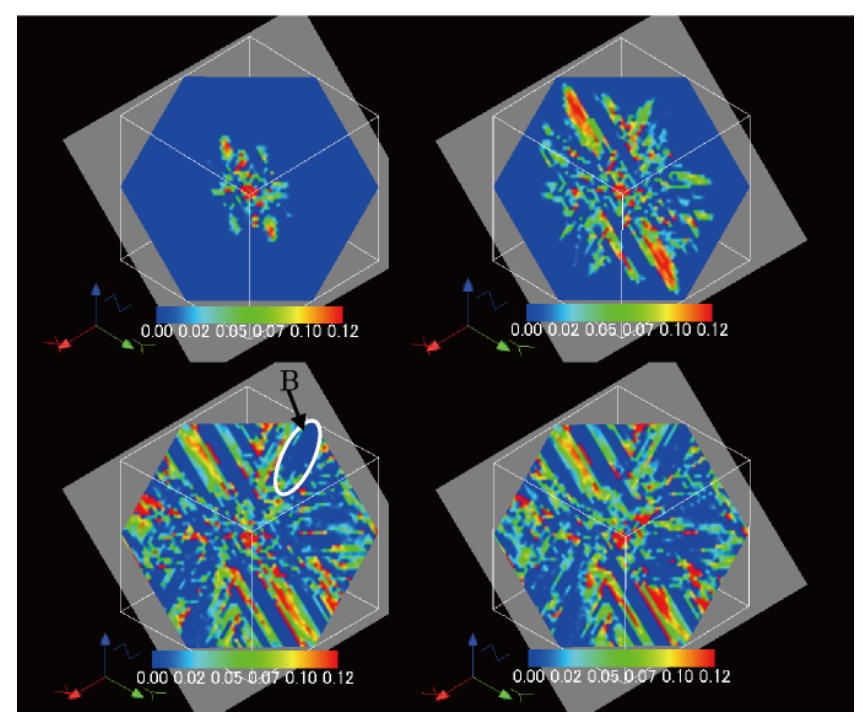

Fig. 13 Time evolution for the plastic strain, $p_{i}^{\alpha}(\mathbf{r}),(i=1,2,3)$, along the $[\overline{101}](101)_{\alpha^{\prime}}$ slip system in view of the (111) plane for (a) $t^{*}=2$, (b) $t^{*}=$ 4 , (c) $t^{*}=8$, and (d) $t^{*}=20$ by phase-field simulation.

the elastic strain energy is small (Fig. 14 (a)) and only exists in a small area near the martensite nucleus located in the center. With the evolution of time, the elastic strain energy increases, which is distributed over the entire region. A comparison of Fig. 14 with Figs. 12 and 13 reveals that the slip deformation also determines the trend of the increase and distribution of elastic strain energy. The values of elastic strain energy shown in Fig. 14 represent the local elastic strain energy in a CP group. By integrating the local values over the entire computational domain, the maximum value of the elastic strain energy should be equal to the value of the elastic strain energy at the completion of martensitic transformation (Fig. 9). Using the slip deformations along the two slip systems, the large strain induced by phase transformation is released, thereby minimizing the total free energy. Hence, the full martensite phase is well presented. 
Figure 15 shows the growth of lath martensite in a 3D space. The cubic skeleton represents the prior austenite lattice. The size of the martensite phase increases around the initial lath martensite nucleus and becomes full martensite at $t^{*}=20$, occupying the entire austenite cube (Fig. 15(d)). Figure 16 shows the time evolution of the lath martensite in 3D space, as can be observed from the outside of the austenite cube. A dark blue cube represents the austenite phase. The six colored areas on the cubic surface correspond to the six lath variants. At $t^{*}=2$ and 4 , the lath martensite is only present inside the austenite cube (Figs. 16 (a) and (b)). However, with the progression of martensitic transition, the lath martensite phase reaches the austenite cubic surface, which can be observed from the outside of the austenite cube (Fig. 16 (c)). With the completion of martensitic transformation, at $t^{*}=20$, the lath martensite phase spreads all over the austenite cubic surface (Fig. 16 (d))

In the above simulation, the Lame constant is used as an isotropic material. The elastic constants of the martensite phase cannot be experimentally estimated because it is impossible to make a single crystal of martensite. In fact, to the best of our knowledge, no studies have reported elastic constants $C_{k l m n}$ of martensite. Then, using the elastic constants calculated by the first principle calculation ${ }^{54)}$, a similar simulation is carried out for a tetragonal martensite crystal. As a result, the block size decreased slightly, but the morphological feature is substantially similar to that in Fig. $16^{55)}$.

\subsection{Dislocation density of lath martensite}

As mentioned above, the morphology of the lath martensite can be characterized by the plastic deformation accompanying dislocation slips, indicating that the maximum dislocation density is evaluated from the plastic strain caused by martensitic transformation. During the transformation, the dislocation density is considered to involve dislocations existing in the austenite phase around the martensite phase because if plastic strain arises in the austenite phase around

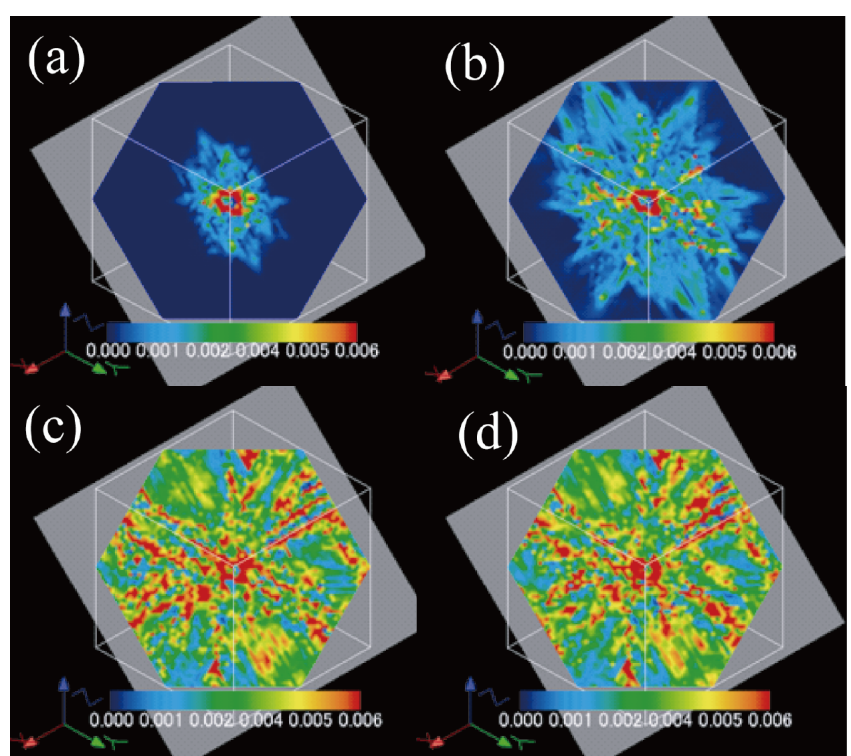

Fig. 14 Time evolution for the elastic strain energy in view of the (111) plane for (a) $t^{*}=2$, (b) $t^{*}=4$, (c) $t^{*}=8$, and (d) $t^{*}=20$ simulated by the phase-field model. the transformed martensite phase, the constraint force from the surrounding austenite phase to the newly formed martensite phase decreases, thereby decreasing the plastic strain in the newly formed martensite phase ${ }^{56}$. In other words, the total strain caused by martensitic transformation corresponds to the value in Fig. 9, and if a part of the strain is covered by the austenite phase, the amount of strain absorbed by the martensite phase is considered to decrease.

To evaluate the maximum dislocation density based on PF simulation of martensitic transformation, the average plastic strain $\left(p_{\text {ave }}\right)$ caused by the transformation is expressed as ${ }^{57)}$

$$
p_{\text {ave }}=\frac{|\mathbf{b}|}{D},
$$

where $\mathbf{b}$ is the Burgers vector and $D$ is the average distance between the neighboring slip planes. The number of lattice

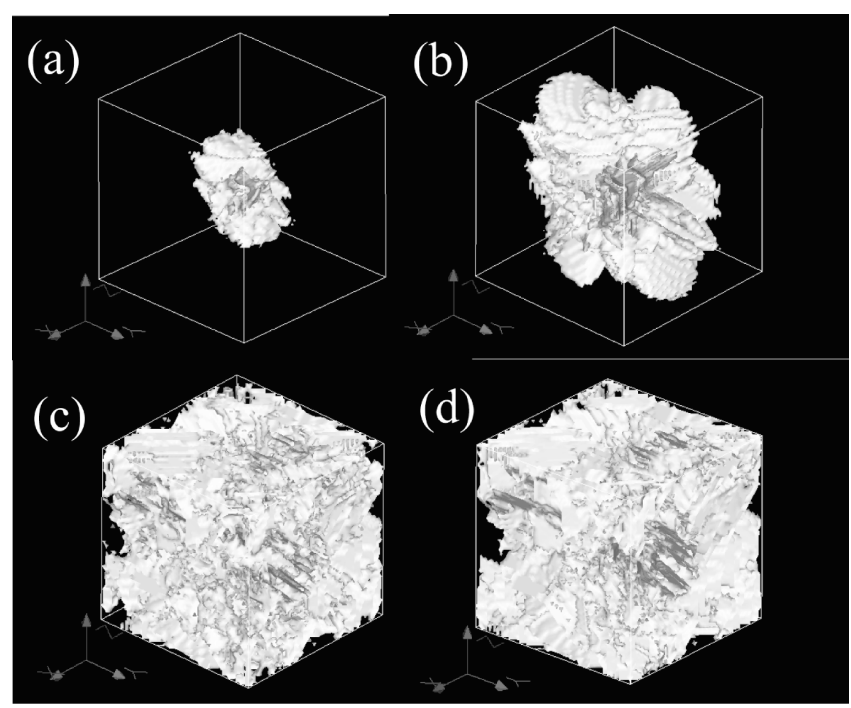

Fig. 15 Growth process of lath martensite at (a) $t^{*}=2$, (b) $t^{*}=4$, (c) $t^{*}=$ 8 , and (d) $t^{*}=20$ in a $3 \mathrm{D}$ space simulated by the phase-field model.

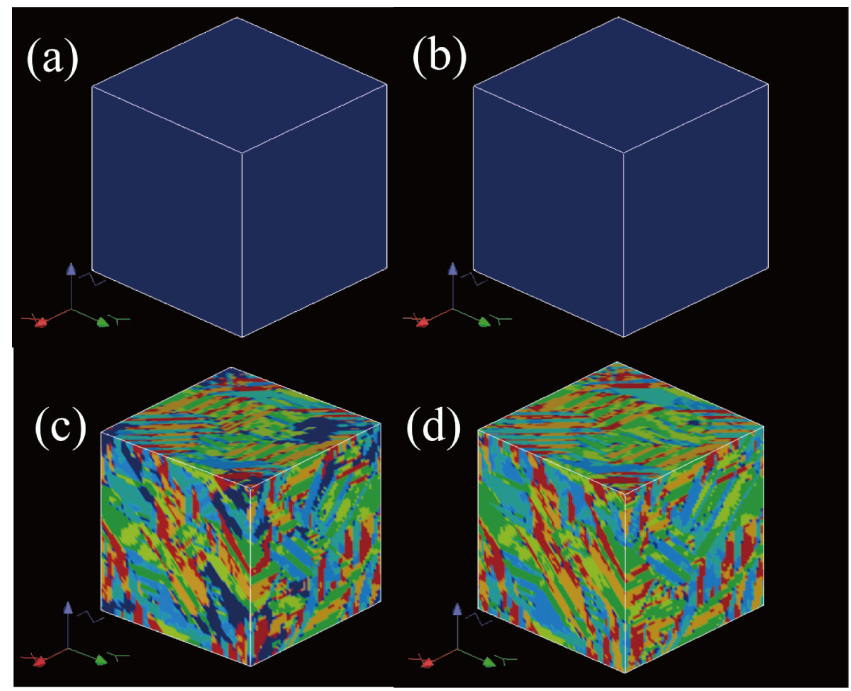

Fig. 16 Time evolution for the lath martensite at (a) $t^{*}=2$, (b) $t^{*}=4$, (c) $t^{*}=8$, and (d) $t^{*}=20$ in the 3D space observed from the outside of the austenite cube simulated by the phase-field model. Each color represents a block variant. 
planes between the two adjacent slip planes for each slip system used in the TTSD model is $m$, and the $D$ value is expressed as

$$
D=m \times d_{h k l},
$$

where $d_{h k l}$ is the distance between the $(h k l)_{\alpha^{\prime}}$ planes. Here, the distance between the neighboring dislocations is approximately related to the maximum dislocation density $\left(\rho_{\mathrm{lim}}\right)$ as $D \approx 1 / \sqrt{\rho_{\text {lim }}}$.

As shown in Fig. 17, the $p_{\text {ave }}$ value is approximately 0.035 when martensitic transformation is accomplished at $t^{*}=20$. Using the value of $\left|\mathbf{b}_{(101) \alpha^{\prime}}\right|=4.06 \times 10^{-10} \mathrm{~m}$ for pure iron, and substituting the $D_{(101)}$ obtained from eq. (47) and $d_{(101)_{\alpha^{\prime}}}$ value as $2.03 \times 10^{-10} \mathrm{~m}$ into eq. (48), the $m_{(101)}$ value is estimated to be 57. Assuming that each of the Burgers vector of $[101]_{\alpha^{\prime}}$ or $[\overline{1} 01]_{\alpha^{\prime}}$ in the TTSD model is composed of two sets of $\langle 111\rangle_{\alpha^{\prime}}$ Burgers vectors (Fig. 5), $m_{(111)}$ should be 114 for the actual slip systems in the martensite phase. Hence, the maximum dislocation density in Fe- $0.1 \mathrm{C}$ steel is evaluated as $2.8 \times 10^{15} \mathrm{~m}^{-2}$.

Concerning the dislocation density in the martensite phase, some data have been reported ${ }^{58)}$ but only a few studies have been reported for low carbon (less than $0.1 \% \mathrm{C}$ ) steels, which is important for heat-resistant steels. Thus, the dislocation density of low-carbon steels is measured by X-ray diffraction (XRD) $)^{59,60)}$, and the results ${ }^{61,62)}$ are compared with the simulation results mentioned above.

A series of experiments is carried out using four kinds of $10 \mathrm{Cr}-5 \mathrm{~W}$ commercial steels containing $0.02,0.03,0.09$, and $0.13 \% \mathrm{C}^{62)}$. The solid line in Fig. 18 represents the average dislocation density for each steel sample. For comparison, the results obtained from TEM using carbon steels are also denoted by a broken line. Because of different steel compositions, it is not possible to directly compare the data denoted by the solid and broken lines, but the dislocation density obtained from TEM is greater than that obtained from XRD at a low carbon content range by a factor of 1.3-1.5. However, the order of magnitude for the dislocation density is $10^{14} \mathrm{~m}^{-2}$ by both methods, with a similar trend of change in the dislocation with a carbon content of up to $0.13 \% \mathrm{C}$. The volume strain of the martensite phase is considered to increase with the carbon content, leading to an increase in dislocation density.

By the comparison of the experimental results with the simulation results $\left(\rho=2.8 \times 10^{15} \mathrm{~m}^{-2}\right)$, the simulation value is greater than the experimental value by a factor of 1.5 . Here, during martensitic transformation, several dislocations contribute to the formation of lath martensite. However, after the phase transition, some dislocations are present in the martensite crystal, which can be observed through experiments. On the other hand, some dislocations pass through out of the martensite crystal, which are possibly used for forming the lath boundaries. Therefore, the simulation result is quantitatively consistent with that obtained from the experiments.

\subsection{Sub-block structure}

As mentioned in Section 1, Morito et al. confirmed the existence of a sub-block structure using an effective experimental tool of EBSD. In this structure, blocks are composed of not just one variant but rather a combination of two specific crystallographic variants as shown in Fig. 19, resembling the lath structure. The formation of the sub-block structure is clearly understood by the Bain group and the type of slip deformation (Fig. 8) that release the strain caused by Bain lattice deformation, as mentioned in Section 2.2. In fact, the simulation results presented in Section 3.2 confirm that the sub-block characteristics are similar to those observed experimentally (Fig. 11).

\subsection{Presence of lath in a block}

With respect to the hierarchical structure, including packets, blocks, and laths in lath martensite, the formation of a block structure comprising 24 variants in a packet is completely understood by the crystal orientation relationship between the matrix austenite phase and the bct martensite phase (Table 1). In contrast, although laths are experimentally observed in a block as regions with fluctuating crystallographic orientations, studies have not reported the formation mechanism even though the term in "lath" is used to characterize a martensite structure. The difference in crystallographic orientation between the laths is so marginal that the presence of lath can be barely distinguished by TEM; hence, the lath and block are sometimes confused experi-

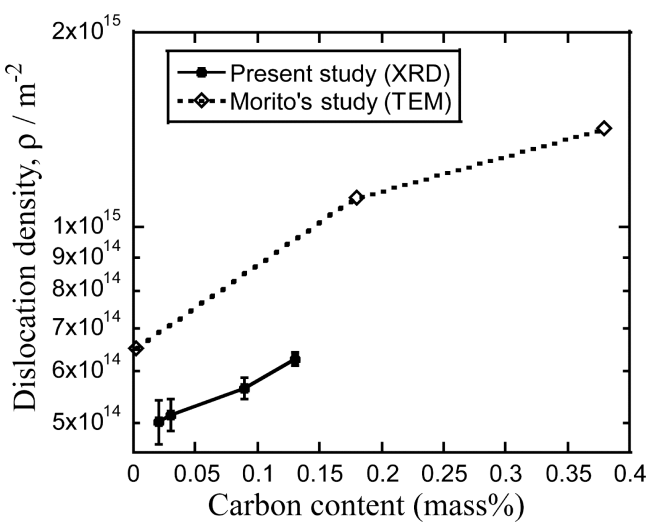

Fig. 18 Change in dislocation density with the content of carbon in low-carbon $10 \mathrm{Cr}-5 \mathrm{~W}$ steels. Solid squares represent the XRD results from this study, while the clear diamonds represent the TEM results reported by Morito et al.
Fig. 17 Simulation result for the the time evolution of the average value of the plastic strain $p_{a v r}$. 


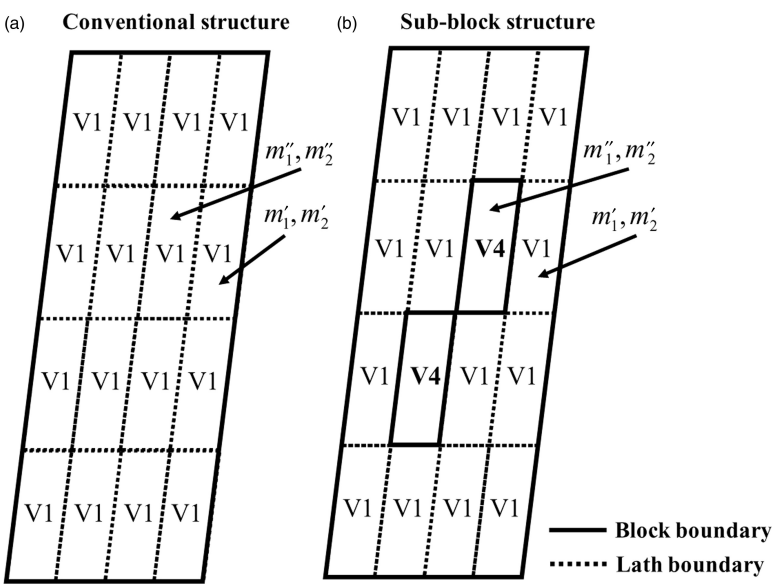

Fig. 19 Schematic of the sub-block structure originating from the value of the slip parameter in the TTSD model.

mentally with respect to size and width.

In contrast to the above situation, as mentioned in Section 2 , the TTSD model reasonably explains the formation and inevitability of lath. For example, in Fig. 8, when a block region (V1: red line) is decided by the condition of $m_{1}>17.8$, each single lath crystal is the region with a unique $m_{1}$ value because it exhibits a degree of freedom within a variant (Fig. 8). The region in one variant being released by a unique slip deformation (unique $m_{1}$ value) is reasonably considered to be the lath. In fact, the crystallographic deviation in the region with $17.8<m_{1}<100$ indicated by a red line is approximately less than $5^{\circ}$, whose value is consistent with the experimental fact that laths are slightly misoriented crystallographically with respect to each other. Thus, as can be schematically observed in Fig. 20, each block indicated by $m_{1}$ or $m_{2}$ contains plural laths represented by $m_{1}^{(i)}$ and $m_{2}^{(i)}$, which are misoriented with respect to each other.

As mentioned above, the TTSD model can provide a clear explanation for the formation mechanism of lath. In addition, this model can reasonably and consistently explain the microstructural phenomena observed experimentally in lath martensite.

In the simulation results in Section 3.2, the existence of blocks V1-V6 is clearly expressed, but the laths in a block have not been expressed at the present time. To present the laths, it is necessary to distinguish the region with a unique $m_{1}$ value in a block. In principle, on the basis of eqs. (47) and (48), the amount of slip deformation is possibly evaluated using the plastic strain, as shown in Figs. 12 and 13, and then the region having a similar amount of slip deformation is divided by histogram segmentation, but this will be reported in a future study.

\section{Summary}

In this study, experimental phenomena and PTMC are reviewed, and the TTSD model based on the experimental facts is described in detail.

The TTSD model is based on the experimental facts that have been reported previously, and is constructed via the approach of the formation mechanism of lath martensite by

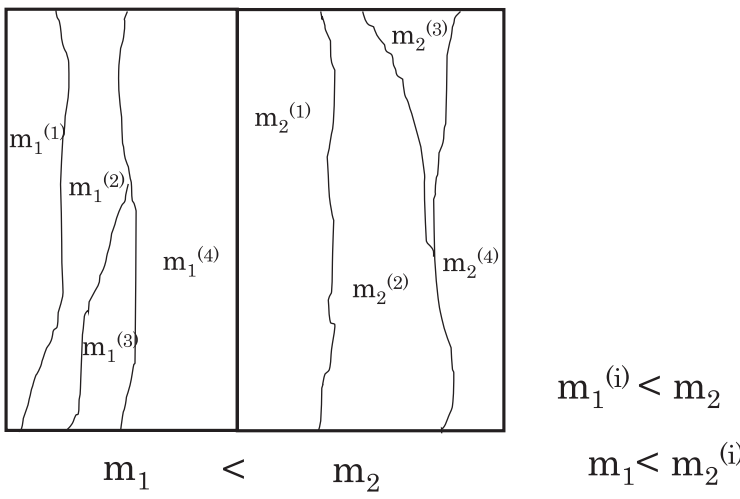

Fig. 20 Schematic of the laths in a block structure originating from the value of the slip parameter in the TTSD model.

solving an inverse problem. We do not try to explain the complex hierarchical structure of lath martensite phenomenologically, but want to make clear which factor and mechanism decide the formation of lath martensite. In particular, without the preliminary introduction of lattice rotation as PTMC, a consistent theory (model) that produces the habit plane by slip deformations (as it turns out, the transformation matrix of eq. (25) involves lattice rotation) is constructed. Although this model focuses on the growth process of lath martensite and does not treat lath nucleation, it may not be a comprehensive model for explaining the formation of lath martensite. However, the phenomena observed in the lath martensite, including (1) the inevitability of the hierarchical structure, (2) sub-block formation, and (3) the meaning (definition) and inevitability of lath existing in a block, can be explained reasonably.

Here, the formation mechanism based on slip deformation is considered because the content of carbon is approximately $0.1 \%$ in heat resistant steels, which we have investigated. Hence, the martensite phase is lath martensite including only dislocations. In the martensite phase, with increasing carbon content, lattice defects are known to change from dislocations to twins. This transition is considered to occur because of the mobility of dislocations. As the formation of twinned crystals needs considerably greater energy compared to the dislocation slips for the general deformation of metal, the metal itself want to deform by the slips. In other words, if slip deformation is possible, twin deformation may not occur. In this sense, the transition from slip deformation to twin deformation in the martensite phase is related to dislocation mobility (such as the Pierce force depending on the carbon content) in martensite itself; hence, the effect of the surrounding austenite phase may not be considered to play an important role.

Finally, even though an academic approach predicts the habit plane of $(h k l)$, the close-packed orientation of [hkl], and the direction of close-packed plane parallel to $[h k l]$, their relationships are the results compromised to each other in the real materials, as shown in Fig. 8, This compromising gives the results for the formation of the habit plane and a slightly deviated direction for the close-packed plane. The stable state may need to be evaluated from the viewpoint of the total energy of the system with respect to whether the 
habit plane, close-packed plane parallel, and close-packed directions are stable. However, the actual microstructure is not necessarily the energy-minimum state, and the martensite phase itself is a metastable phase. Therefore, the total energy of the actual microstructure is considered to fluctuate near the energy-minimum. Thus, it can be said that the actual microstructure is a result reflecting this fluctuated energy state.

\section{Acknowledgements}

I wish to express my gratitude to Professor T. Koyama and Associate Professor Y. Tsukada of Nagoya University for their useful discussion; in particular, they suggest considerably the progression from TTSD model to phase field simulation of the martensite phase. Professor H. Numakura of Osaka Prefectural University gave us the elastic constants of the martensite phase which are calculated by the first principle calculation. The TTSD model was constructed by brainstorming with Mr K. Iwashita, a graduate student (Present: High school teacher in Saitama Prefecture). We also thank Messrs. K. Itoh (Present: NGK Spark Plug Co., Ltd.), F. Ozone, K. Yoshida (Present: Hitachi Construction Machinery Co., Ltd.), and Dr. Z. Cong (Present: NanJin Tech. Univ.), graduate students of Nagoya University, for their help in the simulation work. Furthermore, I am grateful to Dr. T. Kunieda (Present: Nippon Steel and Sumitomo Metal Corp.), Mr. K. Akada (Honda R\&D Co., Ltd.) and T. Shintani (Mitsubishi Heavy Industries, Ltd.), graduate students of Nagoya University, for their help in the experimental work of X-ray diffraction for obtaining dislocation density. This work was partly supported by a Grant-in-Aid for Scientific Research of Japan Society for the Promotion of Science (JSPS), Japan.

\section{REFERENCES}

1) I. Tamura, H. Izumi and S. Isa: Tekko Zairyo Gaku, (Asakura Publishing Co., Ltd., Tokyo, Japan, 1981).

2) T. Maki: Tekko-no-Soshiki Seigyo, (Uchida Rokakuho Publishing Co., Ltd., Tokyo, Japan, 2015).

3) T. Maki and I. Tamura: Tetsu-to-Hagané 67 (1981) 852-866.

4) T. Maki and I. Tamura: Materia Japan 23 (1984) 229-237.

5) T. Maki: Materia Japan 48 (2009) 206-211.

6) T. Maki: Materia Japan 54 (2015) 557-563.

7) T. Maki: Materia Japan 54 (2015) 626-632.

8) T. Maki, K. Tsuzaki and I. Tamura: Tetsu-to-Hagané 65 (1979) 515-524.

9) T. Furuhara: Ferrum 12 (2007) 748-752.

10) T. Furuhara: Ferrum 14 (2009) 97-102.

11) G. Miyamoto, T. Kaneshita, T. Chiba and T. Furuhara: J. Jpn. Inst. Met. Mater. 79 (2015) 339-347.

12) J.M. Marder and A.R. Marder: Trans. ASM 62 (1969) 1-10.

13) S. Matsuda, T. Inoue, H. Mimura and Y. Okamura: Proc. Int. Sympo. on Toward Improved Ductility and Toughness, Kyoto, (1971), 45-66.

14) K. Wakasa and C.W. Wayman: Acta Metall. 29 (1981) 973-990.

15) K. Wakasa and C.W. Wayman: Acta Metall. 29 (1981) 991-1011.

16) S. Morito, H. Tanaka, R. Konishi, T. Furuhara and T. Maki: Acta Mater. 51 (2003) 1789-1799.

17) H. Kitahara, R. Ueji, N. Tsuji and Y. Minamino: Acta Mater. 54 (2006) 1279-1288.
18) G.V. Kurdjumov and G. Sachs: Z. Phys. 64 (1930) 325-343.

19) Z. Nishiyama: Martensitic Transformation, Maruzen(1971)

20) V.I. Izotov: Phys. Met. Metallogr. 34 (1972) 112-120.

21) A.R. Marder and G. Krauss: Trans. ASM 62 (1969) 957-964.

22) S. Morito, H. Huang, T. Furuhara, T. Maki and N. Hansen: Acta Mater. 54 (2006) 5323-5331.

23) J.S. Bowles and J.K. MacKenzie: Acta Metall. 2 (1954) 129-137.

24) J.S. Bowles and J.K. MacKenzie: Acta Metall. 2 (1954) 138-147.

25) J.S. Bowles and J.K. MacKenzie: Acta Metall. 2 (1954) 224-234.

26) M.S. Wechsler, D.S. Lieberman and T.A. Read: Trans. Metall. AIME 197 (1953) 1503-1515.

27) B.P.J. Sandvik and C.M. Wayman: Metall. Trans., A, Phys. Metall. Mater. Sci. 14A (1983) 835-844.

28) P.M. Kelly: Mater. Trans., JIM 33 (1992) 235-242.

29) G.B. Olson and M. Cohen: Metall. Trans. 7A (1976) 1905-1914.

30) G.B. Olson and M. Cohen: Annu. Rev. Mater. Sci. 11 (1981) 1-30.

31) K. Shimizu, M. Oka and C.M. Wayman: Acta Metall. 18 (1970) 1005-1011.

32) A. G. Khachaturyan: Theory of Structural Transformations in Solids, New York. Wiley (1983).

33) K. Iwashita, Y. Murata, Y. Tsukada and T. Koyama: Philos. Mag. 91 (2011) 4495-4513.

34) M. Kato: Mater. Trans., JIM 33 (1992) 89-96.

35) O.D. Sherby, J. Wadsworth, D.R. Lesuer and C.K. Syn: Mater. Trans. 49 (2008) 2016-2027.

36) C.S. Roberts: Trans. AIME 197 (1953) 203-204.

37) B.P.J. Sandvik and C.M. Wayman: Metall. Trans., A, Phys. Metall. Mater. Sci. 14A (1983) 809-822.

38) J. W. Christian: The Theory of Transformations in Metals and Alloys, Pergamon, Oxford (1965).

39) L. Qi, A.G. Khachaturyan and J.W. Morris, Jr.: Acta Mater. 76 (2014) 23-39.

40) P.M. Kelly: Acta Metall. Mater. 38 (1990) 1075-1081.

41) G. Miyamoto, N. Takayama and T. Furuhara: Scr. Mater. 60 (2009) 1113-1116.

42) G. Miyamoto: Materia Japan 49 (2010) 332-336.

43) Y.U. Wang, Y.M. Jin, A.M. Cuitino and A.G. Khachaturyan: Acta Mater. 49 (2001) 1847-1857.

44) A. Artemev, Y.M. Jin and A.G. Khachaturyan: Acta Mater. 49 (2001) 1165-1177.

45) Y.M. Jin, A. Artemev and A.G. Khachaturyan: Acta Mater. 49 (2001) 2309-2320.

46) J.W. Cahn and J.E. Hilliard: J. Chem. Phys. 28 (1958) 258-267.

47) Y.U. Wang and A.G. Khachaturyan: Mater. Sci. Eng. A 438-430 (2006) 55-63.

48) C. Shen and Y. Wang: Acta Mater. 51 (2003) 2595-2610.

49) N. Zhou, C. Shen, M. Mills and Y. Wang: Philos. Mag. 90 (2010) 405-436.

50) S.M. Allen and J.W. Cahn: Acta Metall. 27 (1979) 1085-1095.

51) Z. Cong, Y. Murata, Y. Tsukada and T. Koyama: Mater. Trans. 53 (2012) 1822-1825.

52) Z. Cong, Y. Murata, Y. Tsukada and T. Koyama: Philos. Mag. 93 (2013) 1739-1747

53) Metals data book: ed. by Japan Institute of Metals 3rd ed., Tokyo, Maruzen (1993).

54) M. Souissi and H. Numakura: ISIJ Int. 55 (2015) 1512-1521.

55) K. Yoshida, Y. Murata, Y. Tsukada, T. Koyama, S. Souissi and H. Numakura: CAMP-ISIJ 28 (2015) 251.

56) Y. Tsukada, Y. Kojima, T. Koyama and Y. Murata: ISIJ Int. 55 (2015) 2455-2462.

57) Z. Cong, Y. Murata, Y. Tsukada and T. Koyama: Mater. Trans. 53 (2012) 1598-1603.

58) S. Morito, J. Nishikawa and T. Maki: ISIJ Int. 43 (2003) 1475-1477.

59) T. Unga'r, J. Gubicza, G. Riba'rik and A. Borbe'ly: J. Appl. Crystallogr. 34 (2001) 298-310.

60) T. Shintani and Y. Murata: Acta Mater. 59 (2011) 4314-4322.

61) T. Kunieda, M. Nakai, Y. Murata, T. Koyama and M. Morinaga: ISIJ Int. 45 (2005) 1909-1914.

62) Z. Cong and Y. Murata: Mater. Trans. 52 (2011) 2151-2154. 